der Schuß aus einer Kanone? Muß man nicht demnach die Mechanik ebenso wie die Elektrodynamik von Grund aus abändern und sie auf quantentheoretischer Basis neu aufbauen?

Alle diese Probleme harren noch der Lösung. Aber wie diese endgültige Lösung auch ausfallen mag, so wird sie sich jedenfalls auf das ungeheure vorliegende empirische und theoretische Material zu stützen haben. Noch fehlt der leitende Gedanke, der das Zerstreute sammelt, das Disparate zusammenfabt. Aber wir wollen hoffen, da $B$ der Tag nicht mehr allzufern ist, an dem die gewaltige Vorarbeit, die die heutige Generation geleistet hat, durch die Vollendung der Theorie gekrönt wird.

\section{Anwendungen der Quantenlehre in der Theorie der Serienspektren.}

\author{
Von Dr. Paul S. Epstein, München.
}

\section{Abschnitt.}

Grundlagen.

$\$ 1$. In den. Jahren 1896 bis 1902 hatte Max Planck seine ganze Arbeitskraft in den Dienst der Theorie der Wärmestrahlung gestellt. Mit einer beispiellosen Folgerichtigkeit und Energie schuf er zunächst in einer Reihe von Abhandlungen die begrifflichen Grundlagen für eine solche Theorie, und gelangte um die Wende des Jahrhunderts zur Uberzeugung, daß die gewöhnliche Mechanik und Maxwell-Lorentzsche Elektrodynamik zur Begründung der Strahlungslehre nicht ausreichen. Die konsequente Anwendung dieser klassischen Grundlagen führte nämlich unter allen Umständen auf ein Gesetz der Strahlungsverteilung (das sogenannte Rayleigh-Jeanssche), welches sich im kurzwelligen Gebiete in eklatantem Widerspruch mit der Erfahrung befindet. Hieraus leitete Planck die Notwendigkeit $a b$, in die Strahlungstheorie einen neuen, der Mechanito und Elektrodynamit fremden Gedanken einzuführen. Im Jahre 1901 gelang es ihm auch, mit kühnem Griff das fehlende Glied in die Kette seiner Deduktionen einzufügen. Es war dies die Quantenhypothese, welche nicht nur alle Rätsel in der Theorie der Wärmestrahlung auflöste, sondern, wie sich später zeigen sollte, auch alle übrigen atomistischen Vorgänge beherrscht.

Die Anwendungen der Quantentheorie auf die Atomistik haben in den letzten Jahren zu einer Reihe groBer Erfolge geführt; und auch an dieser neuesten Entwicklung ist Planck in hervorragendem Maße beteiligt. Es ist der Zweck dieses Aufsatzes, eine Ưbersicht über die neueren Ergebnisse zu entwerfen, soweit sie sich auf die Theorie spektraler Serien beziehen. Dementsprechend werden zunächst die begrifflichen Grundlagen der Planckschen Quantenlehre in Kürze erörtert (\$S 2-4) und das nötige Tatsachenmaterial aus der Atomistik und Spektroskopie zusammengestellt $(\$ \$ 5$, 6). Der zweite Abschnitt enthält die ersten er- folgreichen Anwendungen der Quantentheorio auf das Atom, welche sich an den Namen Niels Bohr knüpfen und in der Erklärung der einfachsten Spektralserien gipfeln $(\$ \$ 7-10)$. 'Im dritten Abschnitt findet man die Anwendungen auf Systeme von mehreren Freiheitsgraden, wie sie durch von Planck und Sommerfeld neu geschaffene begriffliehe Hilfsmittel ermöglicht wurde: im wesentlichen die Theorie der Feinstrukturen wasserstoffühnlicher Linien und des Starkeffekts (\&\& 11-15). Den Schlnß bildet eine Erörterung der Anschauungen von Planck über die Struktur des Phasenraumes.

8 2. Hypothese der Energiequanten. - Um den Inhalt der Quantenhypothese klarzumachen. wollen wir das in der Einleitung Gesagte näher ausführen und an den Begriff des ,linearen Resonators" anknüpfen. Wir können uns darunter ein Elektron ${ }^{1}$ ) vorstellen, welches quasielastisch (d. h. durch eine der Entfernung $x$ proportionale Kraft) an eine Ruhelage gebunden ist, und demzufolge sinusartige Schwingungen von einer konstanten, für den Resonator charakteristischen Schwingungszahl $v$ pro Sekunde um dieselbe ausführt:

$$
x=x_{0} \sin 2 \pi v t \ldots . . . . \text {. } 1
$$

Hier bedeutet $t$ die Zeit und $x_{0}$ die Amplitude.

Nach den Gesetzen der Elektrodynamik muk ein solcher linearer Resonator elektromagnetische Wellen von derselben Schwingungszahl $v$ aussenden, und umgekehrt unter der Wirkung einfallender Wellen erzwungene Schwingungen ausführen. Hat man also in einem vollkommen spiegelnden Hohlraum eine gröhere Menge von Resonatoren mit allen möglichen $v$, so müßten sie sich mit der Strahlung und gegenseitig in ein bewegliches Gleichgewicht setzen, bei welchem jede Gruppe von Resonatoren (von einer bestimmten Schwingungszahl v) genau so viel Energie ausstrahlt, als sie von der zugestrahlten Energie absorbiert. Diese Gleichgewichtsstrahlung bezeichnet man als ,schwarze Strahlung und das Problem, welches sich Planck gestellt hatte, bestand darin, die spektrale Energieverteilung der schwarzen Strahlung abzuleiten. Es zeigte sich nun, daR man unfehlbar auf ein falsches Gesetz geführt wird, wenn man voraussetzt, das'ein Resonator bei der Wechselwirkung alle möglichen Energieinhalte (bzw. Amplituden) annehmen kann. Die neue, außerordentlich kiihne Annahme von Planck bestand darin, daß die Energie A eines Resonators ein atomistisches Terhalten besitzt

1) Nach den neueren Forschungsergebnissen hat der Träger der elektrischen Erscheinungen. die sogenannte .Elektrizitätsmenge" Struktur. Die Atome der Elektrizitätsmenge bezeichnet man als Elektronen. Das Elektron hat eine 1844 . mal kleinere Masse $\mu$ als das Wasserstoffatom, seine elektrische Ladung (,Elementareinheit der Elektrizitätsmenge ${ }^{(4)}$ beträgt $e=4,77 \cdot 10^{-10}$ el. st. Einb. Das Verhältnis von Ladung zu Masse ist $\frac{e}{\mu}=5,291 \cdot 10^{17}$ el. st. Einh. 
und ein ganzes Vielfaches einer elementaren Menge $\varepsilon$ sein $m u p$ :

$$
A=n \varepsilon_{3}
$$

wenn $n$ eine ganze Zahl bedeutet. Aus thermodym namischen Gründen (Wiensches Verschiebungsgesetz) darf jedoch die elementare Energie $\varepsilon$ nicht von der Schwingungszahl unabhängig, sonder mul derselben proportional sein : $\varepsilon=h v$. Als Ausdruck der ersten Fassung der Planckschen Hypothese, die man als ,Hypothese der Energiequanten $^{\prime \prime}$ bezeichnet, gewinnt man daher die Formel

$$
A=n h \text { v. . . . . . . . . (2 }
$$

Hier ist $h$ eine universelle Konstaate von der Dimension einer Wirkung (Energie $\times$ Zeit), welche daher als das ,Plancksche Wirkungsquantum" bezeichnet wird. Aus den Strahlungsmessungen von Kurlbaum, Lummer und Pringsheim erhielt Planck den numerischen Wert

$$
h=6,55 \cdot 10^{-2 "} \text { erg. sec. }
$$

Den Ausdruck für die Energie $A$ des linearen Resonators erhält man, wenn man bedenkt, daß im Momente des Durchgangs durch den Nullpunkt $(t=0)$ nur kinetische Energie vorhanden ist. Ist $\mu$ die Masse des Elektrons:

$$
\left.A=\frac{\mu}{2}(\dot{x})_{t=0}^{2}=2 \mu\left(\pi \vee x_{0}\right)^{2} .^{2}\right)
$$

Beziehung (2) liefiert daher

$$
\left.2 \pi^{2} \mu v x_{0}{ }^{2}=n h . . . . .3\right)
$$

Es sind also nur bestimmte ausgewählte Amplituden $x_{0}$, nämlich diejenigen, welche der Formel (3) genügen, mit der Quantenhypothese verträglich.

\$3. Hypothese der Wirkungsquanten. Später gab Plancle seiner Hypothese eine andere Fassung, welche klarer hervortreten läBt, das die Wirkung and nicht die Energie das Unveränderliche ist. Dabei macht er Gebrauch vom mechanischep Begriff des Impulses oder der $B e^{-}$ wegungsgröße. Bekanntlich ist bei kartesischen Koordinaten und konservativen Kräten (d. h. Kräften, welche ein Potential besitzen), der einer kartesischen Lagenkoordinate $x$ eines Massen. puntites von der Masse $\mu$ entsprechende Impuls

$$
p_{x}=\mu \dot{x} .
$$

Aber auch unter komplizierteren Verhältnis" sen kann man im allgemeinen die den Lagen koordinaten $q$ eines Systems zugehörigen Impulse $p$ definieren. Dabei ist durch die Kenntnis der Werte aller $p$ und $q$ in irgend einem Augenblick die Bewegung des Systems vollständig und für alle Zeiten bestimmt.

Im besonderen Falle eines einzigen Freiheitsgrades kann man $p$ leicht als Funktion von $q$ angeben. Z. B. hat man für den linearen Resonator nach (1)

$$
\frac{p_{x}}{2 \pi v \mu}=x_{0} \cos 2 \pi v t \ldots . . . . \text {. (4 }
$$

1) Durch einen Punkt wird die Ableitung naeh der Zeit angedeutet: $\dot{x}=a^{z} x / a t$. oder wenn man die Gleichurgen (1) und (4) quadriert und addiert

$$
\left(\frac{x}{x_{0}}\right)^{2}+\left(\frac{p_{x}}{2 \pi v \mu x_{0}}\right)^{2}=1
$$

Das ist bekanntlich die Gleichung einer Ellipse von den Halbachsen $a=x_{0}$ und $b=2 \pi v \mu x_{0}$. Wenn man also $p_{x}$ als Ordinate, $x$ als Abszisse aufträgt (Fig. 1), erhält man für jeden speziellen Wert von $x_{0}$ eine Ellipse, für alle möglichen $x_{0}$ eine Schar ähnlicher konzentrischer Ellipsen. Im allgemeinen Fall eines durch eine Koordinate $q$ und den zugehörigen Impuls $p$ bestimmten Systems wird man durch die analoge Konstruktion eine andere (nicht elliptische) Schar von Kurven erhalten, ron denen jede die Bewegung des Systems für einen speziellen Wert der Energie darstellt.

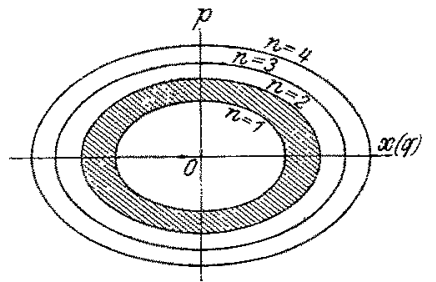

Fig. 1.

Der Planckschen Annahme zufolge sind nach Gleichung (3) nicht alle Kurven der Schar, sondern nur gewisse ausgewählte möglich. Und zwar besteht die spätere Formulierung von Planck darin, daß der Inhalt der Fläche awischen zwei aufeinanderfolgenden quantentheoretisch aulässigen Kurven gleich dem Wirkungsquantum hein soll. Mathematisch formuliert gibt das

oder

$$
\begin{aligned}
& \int d p d q=h . \\
& \left(p_{n}-p_{n-1}\right) d q=h .
\end{aligned}
$$

Die Integration ist über das zwischen den beiden Kurven eingeschlossene, in der Figur für $n=2$ schraffierte Gebiet zu erstrecken, $p_{n}$ und $p_{n-1}$ beziehen sich auf die Werte des Impulses an den Grenzkurven.

Durch Summieren der Ausdrücke

$$
\begin{gathered}
\int\left(p_{1}-p_{0}\right) d q=h, \int\left(p_{2}-p_{1}\right) d q=h, \ldots . . \\
\int\left(p_{n}-p_{n-1}\right) d q=h
\end{gathered}
$$

ergibt sich für die $n$-te quantenmäBig znlässige oder, wie wix sagen wollen, statische Bewegungsform ${ }^{\prime *}$ :

$$
\int\left(p_{n}-p_{0}\right) d q=n h \ldots .(5 a
$$

wenn $p_{0}$ den Impuls an der innersten statischen Kurve von kleinstem zulassigen Flächeninhalt bedeutet. Einen von Null verschiedenen Wert von $p_{0}$ darf man dann erwarten, wenn das betrachtete System aus mechanischen Gründen einen Grenzzustand besitzt, dem eben der Impuls $p_{0}$ entspricht. Beim linearen 'Resonator ist dies nicht der Fall, hier sind auch die kleinsten Amplituden 
und Impulse bis zur-vollständigen Ruhe $\left(p_{0}=0\right)$ mechanisch möglich. Für solche Systeme, welche die Mehrzahl bilden, reduziert sich die Quantenbedingung (5 a) einfach auf

$$
\int p d q=n h
$$

d. h. der von eimer statischen Kurve umschiossene Flacheninhalt ist ein Multiplum des Wirhungsquantums h.

Es ist leicht zu sehen, dab diese Fassung der Planckschen Annahme, welche als ,Hypothese der Wirkungsquanten" bezeichnet wird, für den Fall des linearen Resonators mit der Hypothese der Energiequanten (2) übereinstimmt. In der Tat ist der Inhalt der umschlossenen elliptischen Fläche hier gleich

$$
n h=\pi a b=2 \pi^{2} \vee m x_{0}^{2}
$$

in genauer Utbereinstimmung mit Formel (3).

Einer der Vorteile, welchen diese Formulierung der Quantenhypothese bot, bestand darin, daß es dabei möglich war, die erforderlichen $A b-$ änderungen der Elektrodynamik auf ein Minimum zu reduzieren, wenigstens die Absorption der Energie kontinuierlich zu gestalten und das wesentlich Neue der Auffassung in die Berechnung der statistischen Wahrscheinlichkeiten zu verlegen. Da es jedoch für das Folgende nicht darauf ankommt, ob wir die statischen Bewegungsformen als die einzigen möglichen, oder als nur in statistischem. Sinne ausgezeichnet auffassen, wollen wir auf diese Fragen erst später (in \& 16) zurückkommen.

Als weiteren Vorzug der zweiten Fassung erkennt man den Umstand, daB sie vom Begriff der Schwingungszahl keinen Gebrauch macht und sich daher nicht von vornherein auf periodische Bewegungen beschränkt. In der Tat ist es in der Folge gelungen, sie sowohl auf Systeme von mehreren Freiheitsgraden als auch auf gewisse Klassen nichtperiodischer Bewegungen anzuwonden.

\$ 4. Entwicklung des Gedankens der Energiequanten. - Merkwüdig ist, daB die vom Impulsbegriff ausgehende Auffassung dex Wirkungsquanten den Gedanken der Energiequanten nicht überflüssig gemacht, sondern dessen Anwendung nux an eine andere Stelle verschoben hat. In der Tat wurde dieser Begriff mit Erfolg zur Erklärung mehrerer Erscheinungen herangezogen. Besonders Einstein, dem wir den Ausbau der Quantentheorie nach den verschiedensten Richtungen verdanken, vertrat nachhaltig den Standpunkt, daB sich in den Wirkungen einer Strablung von der Schwingungszahl v das Energiequantum $h v$ äuBern müsse. Auf diesem Wege gab er u. a eine Darstellung der quantitativen Verhälnisse im lichtelektrischen Effelct: das aus einem Metall unter der Wirkung von einfallendem altravioletten Licht (Schwingungszahl v) ausgesandte Elektron erwirbt, aach seiner Hypothese, rom Licht die kinetische Energie $h v$, von der es einen Teil $P$ für die Arbeit aufbraucht, die es $z u$ leisten hat, um durch die Oberfläche hindurchzutreten und den Verband der Metallatome zu verlassen. Es ergibt sich demnach für die Geschwindigkeit, $v$ eines lichtelektrischen Elektrons die Einsteinsche Gleichung (1905)

$$
\frac{\mu v^{2}}{2}+P=h v
$$

welche sich durehaus bostätigt, und nenerdings von. $R$. Millikan (1916) als eine Methode zur genaven Bestimmung des Wertes von $h$ angewandt wurde.

Die Umkehrung dieses Gedankens, die Annahme, daß die bei einem Lichtemissionsvorgang von unbekanntem Mechanismus ausgesandte Schwingungszahl durch die zur Verfügung stehende Energie bestimmt werden könne, finden wir zum ersten Mal bei W. Wien and J. Stark. W. Wien ${ }^{1}$ faBt den Vorgang der Entstehnng von Röntgenstrahlen durch plötzliche Bremsung von Kathodenstrahlen ${ }^{2}$ ) in der Antikathode einer Röntgenröhre ins Auge, und stellt die Fypothese auf, daB die Schwingungszahl ver ausgesandten Röntgenstrahlen durch die kinetische Energie $T$ des gebremsten Elektrons nach der Beziehung $\left.T=c h v^{3}\right) \ldots . . . .(6$ bestimmt wird. Beachtenswert ist bei dieser Auffassung, dab die Größe $h v c$ keineswegs mit der in Form von Röntgenstrahlung emittiexten Energie übereinstimnat, denn letztere beträgt, wie man berechnen kann $^{4}$ ) und sich aus Messungen ergeben hat5), nur einen geringen Bruchteil (von der GröBenordnung $0,2 \%$ ) der Kathodenstrahlenenergie. Bedingung (6) bestimmt also lediglich die Schwingungszahl der ausgesandten Strahlung, ohne etwas über deren Energiemenge auszusagen, weshalb wir diese Gleichung als "Frequenzbedingung" bezeichnen.

Diese Beziehung ist neuerdings (bis zu einer Spannung von 40000 Volt) gepruft worden ${ }^{4}$ ). Es bestätigt sich mit voller Schärfe, daß die größte Schwingungszahl dor Röntgenstrahlen, welche durch Elektronen von einer bestimmten kinetischen Energie angeregt werden, durch Gleichung (6) gegeben ist.

Die erste Anwendung einer ähalichen Uberlegung auf das optische Spektrum geht auf J. Stark ${ }^{\pi}$ zurück. Er betrachtet die Emission der Linien des Quecksilberspektrums und geht von der Annahme aus, daB die Vorbedingung für die

1) W. Wion, Gött. Nachr. S. $098,1907$.

2) Welche bekanntlich aus schnell bewegten Elek tronen bestehen.

a) $c$ bedeutet die Lichtgeschwindigheit. Unter der Schwingungsaahl $y$ verstehen wir hier und im Folgenden, wie dies in der Spektroskopie iblich ist, die reziproke Wellenlänge $v=1 / \lambda$. Genatu genommen ist dies die Zahl der Schwingmgen in 1/c Sekunden.

4) Sommerfeld (Müneh. Ber. \$. 1, 1911) hat in einer Arbeit, die auf die spätere Entwicklumg der Quantentheorie von großern EinfluB war, die Wiensche Theorie modifiziert und quantitativ ausgebaut.

a) W. Wien, Ann. d. Phys. 18, S. 991, 1905.

b) Diane und Hunt, Phys. Rev. 6 , S. 166, 1915.

7) W. Stenting, Phys. Zt. 10, 5. 789. 1909. 
Aussendung derselben das Vorbandensein von ionisierten Atomen (d. h. Atomen, von denen ein Elektron abgetrennt ist) und freier Elektronen im Ilg-Gas sei. Das Leuchten kommt, seiner Meinung nach, durch die Wiederanlagerung eines Elektrons an das ionisierte Atom zustande. Da bei diesem Vorgang nur eine endliche Energjemenge rerfügbar ist, nämlich die Energie $T$, welche max umgekehrt zur Entfemung des Elektrons aus dem Atom ins Unendliche aufwenden muB (die sogenannte Ionisierungsspannung), und welche aus direkten Messungen bekannt ist, schloß Stark, daß die Grenze des Quecksilberspektrums, d. h. die größte Sehwingungszahl $v$, deren Emission möglich ist, dureh die Beziehung (6) bestimmt wird. Neuere Messungen der Ionisierungsspannung des Quecksibers ${ }^{\mathrm{T}}$ ) bestutigen die quantentheoretische Gleichung (6), die Sohwingungszahl $v$, die sich ergibt, bezieht sich jedoch nicht anf die Bandengrenze, sondern auf lie Resonanzstrahlung des Quecksilbexs $(253,6 u \mu)$.

85. Strultur der Serienformeln. Das Ritzsche Kombinationsprinzip. - Unser Zweck ist, eine Übersicht über die Erfolge zu geben, die in den letzten Jahren in der Erklärung der spektralen Gesètze durch Anwendang der Quantentheorie auf die Atomistik erreicht wurden. Daher ist es nötig, zunächst einiges über die Struktur der spektralen Formeln und die modernen Anschauungen rom $\mathrm{Bau}$ der Atome zu sagen.

Beim Studium der Linien, ans denen ein Spektrum besteht, hat man bei gewissen Folgen dieser Linien, welche man als ,Serien" bezeichnet, vin ähnliches Aussehen und gleichmäbiges physikalisches Verhaltén festgestelit und daraus auf ihre genetische Zusammengehörigkeit geschlossen. Die formelmäBige Darstellung spektraler Serien wurde bereits im Jahre 1885 durch eine $\mathrm{Un}_{1-}$ tersuchung von Balmer eingeleitet, der für die später nach ihm benannte Serie des Wasserstoffs die folgende Formel aufstellte:

$$
v=N\left(\frac{1}{2^{2}}-\frac{1}{m^{2}}\right), \quad m=3,4,5 \ldots . . . . \text { ( }
$$

$N$ bedeutet dabei eine Konstante, als deren bester Weit gegenwärtig 109677,69 gilt. Von der Balmerserie, der einzigen Serie, welche der Wasserstoff im sichtbaren Spektrum besitzt, sind 29 Linien bekannt, welche in der Reihenfolge abnehmender Wellenlängen mit $H_{\alpha}, H_{\beta}, H_{\gamma}, H_{\delta}, \ldots$ bezeichnet werden. In den Spektren von Wasserstoffröhren wurden allerdings nur die ersten 13 dieser Linien gefunden, die übrigen entriehen sich der terrestrischen Beohachtung durch ihre Lichtschwäche, sind aber aus Sternspektren bekannt. Formel (7) gibt mit nichts zu wünschen ïbrig lassender Schärfe die Schwingungszahlen sämtlicher 29 Linien, wenn man für $m$ die sukzessiven ganzen Zahlen ron 3 bis 31 einsetzt. Neuere Untersuchungen haben übrigens gezeigt,

1) J. Frank und G. Hertz, Verh, d. D. Phys. Ges. $16,512,1914$ dal die Linien der Balmerserie nicht einfach sind, sondern in mindestens zwei sehr dicht nebeneinanderliegende Komponenten aufgelöst werden können.

Gleichfalls dem Wasserstoff wurde die astronomische Serie zugeschrieben, welche $W$. C. Pichering $1896 \mathrm{im}$ Spektrum des Sterns $\zeta$ Puppis gefunden hatte.

$v=N\left(\frac{1}{2^{2}}-\frac{1}{m^{2}}\right), \quad m=1,5 ; 2,5 ; 3,5 ; \ldots \ldots$

Wir werden sehen ( $\$ 10)$, daB sie in Wirklichkeit dem ionisierten Helium angehört.

Charaktexistisch für die Struktur dieser Formeln ist, dal die Schwingungszahl $v$ hier als Differenz zweier Terme $N / 2^{2}$ und $N / m^{2}$ erschcint. Durch eine eingehende Analyse des damals vorhandenen experimentellen Materials zeigte nun der schwedische Physikex Rydberg im sahre 1890, daf sich viele Seriengesetze bei verschiedenen Stoffen durch die veraligemeinerte Formel

$$
\pm v=\frac{N}{\left(m_{1}+\mu_{1}\right)^{2}}-\frac{N}{\left(m_{2}+\mu_{2}\right)^{2}}
$$

gut darstellen lassen. $N$ ist dabei dieselbe Unveränderliche, welche in dex Balmerschon Forwel (7) anftritt, sie gewinnt also die Bedeutung einer Universalkonstanten und wird als , Pydbergsche Konstante" bezeichnet. $\mu_{1}$ und $\mu_{2}$ sind zwei weitere für das betreffende Element charakteristische Koustanten, $m_{1}$ und $m_{2}$ ganze Zahlen (,Ordnungszahlen"); das Vorzeichen ist so zu wählen, daß $v$ positiv wird. Bei festgehaltenem $m_{1}$ erhält man bereits eine Serio, wenn $m_{2}$ die Reihe der ganzen Zahlen durchläuft. Nimmt man noch die Variabilität ron $m_{1}$ hinzu, so könnea mehrere Serien durch den einzigen Ausdruck (9) dargestellt werden.

Mit zunehmender Genauigkeit der spektralen Messungen erwies sich indessen die Rydbergsche Formel als ungenügend. Die beste der später vorgeschlagenen rührt von W. Ritz her (1903), der unter Beibehaltung der Auffassung von $v$ als Differenz zweier Terme nur jedem der beiden Terme eine veränderte Gestalt gab. Noch größere Verdienste um die Spektroskopie erwarb sich dieser leider jung verstorbene schweizerische Gelehrte durch die Aufstellung seines sogenannten ,Kombinationsprinzips" (1908), durch welches er den Beweis erbrachte, daß die beiden Terme, als deren Differenz, die Schwingungszahl erscheint, keine zufällige Eigenschaft der mathematischen Formulierung sind, sondern unabhängig von dieser einen wirklichen physikalischen Sinn, eine objektive Existenz besitzen.

Dieses Kombinationsprinzip besteht darin, dab man einen der beiden Terme, welche irgend eine Linie einer Serie ergeben, mit einem der Terme, die $\mathrm{zu}$ einer anderen Linie (derselben Serie oder gewisser anderer Serien desselben Elementes) gehören, kombinieren kann, derart, dafs die Differenz wieder eine Spektrallinie ergibt. Auf diese Weise ist es Ritz gelungen, sowohl neue Serien aufzufinden, als auch einige Linien, 
welche auBerhalb des Seriensystems zu stehen schienen, in dasselbe einzuordnen. Z. B. in Anwendung auf die Balmersche Formel (7) hat Ritz die Fxistenz einer Wasserstoffserie ron der Form

$$
v=N\left(\frac{1}{3^{2}}-\frac{1}{m^{2}}\right), \quad m=4,5,6 \ldots .(7 a
$$

vorhergesagt, welche im Ultraroten liegen mub. Dies konnte noch im selben Jahre (1908) durch Messungen von Paschen bestätigt werden, der für Wellenlängen in Luft $(\lambda)$ und Schwingungszahlen $(v)$ der beiden ersten Linien die folgenden Werte angibt:

\begin{tabular}{c|c|c|c|c}
\hline$m$ & $\begin{array}{c}\lambda \text { in A.-E. } \\
\text { beob. }\end{array}$ & ber. & $\begin{array}{c}v \\
\text { beob. }\end{array}$ & ber. \\
\hline 4 & $\begin{array}{l}18751,3 \\
12817,6\end{array}$ & 18751,6 & 5331,58 & 5331,49 \\
5 & 12818,7 & 7799,70 & 7799,10
\end{tabular}

Schon etwas früher wurden von Lyman im Ultravioletten die ersten Glieder der Serie

$$
v=N\left(\frac{1}{1^{2}}-\frac{1}{m^{2}}\right), \quad m=2,3,4 \ldots
$$

gemessen, aus der nach dem Kombinatiousprinzip sowohl die Balmersche als die Ritz-Paschensche Serie hervorgehen.

In der Folge hat besonders Paschen viel mit dem Kombinationsprinzip gearbeitet, ihm haben wix die experimentelle Begründung desselben durch Auffindung und genaue Messung von Kombinationslinien in allen Serienspektren $z u$ verdanken, sowie auch die endgültige Zusammenfassung der verschiedenen Serien eines chemischen Elementes in ein Seriensystem. Vorn Standpunkt dieser Systematik und nach Analogie mit anderen Elementen mïBte man zu der Pickeringschen Serie (8), die man dem Wasserstoff zuschrieh, noch eine andere mit der Formel

$v=N\left(\frac{1}{1,5^{2}}-\frac{1}{m^{2}}\right), \quad m=2,3,4 \ldots$

erwarten, worauf bereits $R y d b e r g$ hingewiesen hatte. Die Grundlinie dieser Serie wurde mit der Linie $v=4687,88$ (ber. $v=4687,90) \mathrm{im}$ Spektrum gewiaser Fixsterne identifiziert, aber erst im Jahre 1912 gelang es Fowler, diese Serie in der terrestrischen Ernission zu beobachten. Im Lichte der elektrischen Entladung in einer mit einem Gemisch von Wasserstoff und Helium gefüliten GeiBlerröhre erhielt er drei Linien der Serie (8), vier der Serie (8a) und drei Linien der ultravioletten Serie

$v=N\left(\frac{1}{1,5^{2}}-\frac{1}{m^{2}}\right), \quad n=2,5 ; 3,5 ; 4,5 ; \ldots$.

Die beobachteten Schwingungszahlen waren um ein Geringes größer als die nach den Formeln $(8 \mathrm{a}, \mathrm{b})$ berechneten. Bemerkenswert ist, daB es unmöglich war, diese Linien in reinem Wasserstoff zu erzeugen, und sich ein Zusatz von Helium als unbedingt nötig erwies.

\$ 6. Das Rutherfordsche Atommodell. - Seit die Physik zu der Erkenntnis gelangt ist, daß elektrische Ladungen innerhalb des Atoms eine wesentliche Rolle spielen, hat es nicht an Versuchen gefenlt, Atommodelle zu konstruieren. Das beliebteste Modell war lange Zeit die von Lord Kelvin (1902) stammende sogenannte, Aepinus kugelrs: Die positive Elektrizität ist kontinuierlich mit konstanter Dichte über das ganze (kugelförmige) Atom ausgebreitet; im Innern dieser Kugel betinden sich Elektronen in solcher Anzahl, dab ihre Gesamtladung gerade die positive Laduag der Kugel neutralisiert. Dies Modell bot den Vorteil, daß die Elektronen in demselben eine statische Gleichgewichtslage haben, so daB man nicht von rornherein ihren. Bewegungszustand in Betracht zu ziehen brauchte. Es blieb allerdings unklar, welche Kräfte die poșitive Elektrizität der Kugel zusammenhalten. Besonders von $J . J$. Thomson wurde dieses Modell weitgehenden atomistischen Betrachtungen zu Grunde gelegt. Von seinen Resultaten ist für uns hauptsächlich das folgende von Wichtigkeit: Die Anzahl $x$ der Eleztronen in einen Atom ist ungefähr gleich der Hälte des Atomgewichts 1 . Von den verschiedenen physikalischen Erscheinungen, welche Thom son übereinstimmend zu diesem. Resultat geführt haben, ist die Zerstreuung von Röntgenstrahlen an verschiedenen Stoffen hervorzuheben: die sich hieran knüpfenden Überlegungen haben nämlich einzig die Annahme, daB im Atom Elektronen vorhanden sind, zur Voraussetzung und sind vou der Art der Bindung derselben unabhängig. Daher bleibt das Thomsonsche Ergebnis über die Elektronenzahl auch für beliebige andere Atommodelle gültig.

Andererseits wurde verschiedentlich mit der Vorstellung gearbeitet, das Atom sei aus diskreten positiven Bestandteilen und Elektronen aufge: baut, welche sich gegenseitig mit Coulombschen Kräften beeinflussen und nach Art eines Planetensystems umeinander bewegen ${ }^{1}$; denn ein statisches Gleichgewicht ist bei Kräften, die mit dem umgekehrten Quadrat der Entfernung wirken. unmöglich. Erst ror wenigen Tahren (1911) hat ein scheinbar unbedeutendes experimentelles Er" gebnis es Rutherford ermöglicht, die Frage zu Gunsten dieser letzteren Klasse von Atommodellen zu entscheiden. Auf seine Veranlassung hatten nämlich Geiger und Marsden (1909) die Ablenkung von $\alpha$-Strahlen ${ }^{2}$ ) von ihrer geradlinigen Bahn beim Durchgang durch dünne Blättchen verschiedener Stoffe untersucht and festgestellt. daß in einem Bruchteil der Fälle auch sehr große (größer als 90') Ablenkungswinkel als Resultat. des ZusammenstoBes der $a$-Partikel mit einem einzigen Atom vorkommen. Damit eine so er-

1) Sehon vor der Entdeckung des Elektrons fabte F. Richarz (1894) das Molekul als System von zwei einander umkreisenden Planeten (positives und negatives Ion anf.

2) Die $\alpha$-Strahlen werden ron radioaktiven Substanzen ausgesandt und bestehen aus mit grober Geschwindigkeit abgeschleuderten. Heliumatomen, welche eine positive Ladung (von gwei Elementareinbeiten) tragen. 
Heft 17.
26.4 .1918 Epstein: Anwendungen der Quantenlehre in der Theorie der Serienspektren.

hebliche Abweichung zustande kommt, mub das x-Teilehen beim. Durchgang durch das Atom eine entsprechend starke (elektrische) Abstoßung "erfahren, und die Diskussion der Verhältnisse im Kelvinschen Modell zeigt, daß die hier vorhandenen elektrisohen Felder für die Erklärung dieses Effektes viel zu gering sind. Genügend starke Feldstärken sind nur dann möglich, wenn die gesamte positive Ladung des Atoms, die ja nach den erwähnten Arbeiten von Thomson schon ungefähr bekannt war, auf einen sehr kleinen Raum, der sogenannten positiven ,Kern k konzentriert ist.

Unter dieser Annahme konnte Rutherford berechnen, dab die Anzahl der um einen Winkel $\varphi$ ron der Geraden abgelenkten $\alpha$-Teilchen den folgenden Größen proportional ist: $1 . \sin ^{-4}(\varphi / 2)$ (oder $p^{-4}$ für kleine $\left.\varphi\right) ; 2$. der Zahl der Atome pro Volumeinheit der zerstreuenden Substanz; 3. der Schichtdicke $d$ der letzteren (solange $d$ klein ist); 4. dem Quadrat der Kernladung $E$; 5. dem umgekehrten Quadrat der kinetischen Fnergie der z-Partikel, Mit Hilfe des 4. Resultats war es möglich, aus dem vorliegenden experimentellen Material die Kernladungen $E \doteq x e$ zu berechner. und es ergab sich in Utbereinstimmung mit Thomson, daß $x$ ungefähr der Hälfte des Atomgewichtes $M$ gleich ist:

$$
x=\frac{M}{2} \quad \ldots . . . . .(10
$$

Durch exreute Versuche von Geiger und Marsden (1913) wurden die Rutherfordschen Folgerungen in allen Einzelheiten geprüft und als mit der Erfahrung übereinstimmend befunden. Die plötzlichen Ablenkungen nach dem Zusammensto $\mathrm{B}$ werden besonders augenfällig in Gasen. Es gelingt hier nämlich nach einer von C. T. R. Wilson angegebenen Methode, den Weg ter $\alpha$-Teilchen sichtbar zu machen und zu photographieren (Fig. 2). Man bemerkt gegen das Ende der sichtbaren Bahn (Reichweite) einzelner Teilchen, wo ihre Geschwindigkeit bereits geschwächt ist, einen mehr oder minder scharfen Knick, welcher eben das Resultat eines besonders zentralen Zusammentreffens mit einem Gasatom ist.

Auf diese Weise gelangt man zur ,Kerntheorie der Atome", die man auch als Sonnentheorie bezeichnen könnte: Das Atom besteht aus einem Kern, in dem die gesamte positive Ladung ( $E$ $=* e$ ) und nahezu die ganze Masse des Atoms konzentriert ist, and einer Wolke von $*$ Elehtronen, welche sich in engeren and weiteren Bahnen nach Art von Planeten um den Kern bewegen. Für das Verhalten eines solchen Sonnensystems gegenüber einem andern, d. h. für die chemischen Eigenschaften des Atoms ist in erster Linie die Verteilung der peripheren Elektronen maBgebend, ebenso wird das optische Spektrum im wesentlichen durch die Peripherie bestimmt. Aber auch von den inneren Elektronen, die sich eng um den Kern bewegen, erhalten wir Kenntnis durch die
Röntgenspektren der Elemente, worauf wir noch in $\$ 14$ zu sprechen kommen.

Die Differenz der Atomgewichte benachbarter Elemente im periodischen System beträgt im Durchschnitt zwei Einheiten, dem entspricht nach der ungetähren Beziehung (10) eine Zunahme der Kernladung \% um eine Elementareinheit beim Lbergang won einem Element zum nächsten. Fs liegt die Auffassung nahe (van den Broek 1913), daP die Stellung im periodischen System nicht durch das Atomgewicht, sondern durch die Kernladung, oder wie man sich vielfach ausdrückt, die Ordnungszahl $\%$ des Elementes bestimmt wird. Man braucht dazu nur anzunehmen, daß durch die Kernladung bereits die ganze Verteilung der Elektronen um den Kern und der Charakter ihrer Bahnen bestimmt ist, was, wie wir gesehen haben,

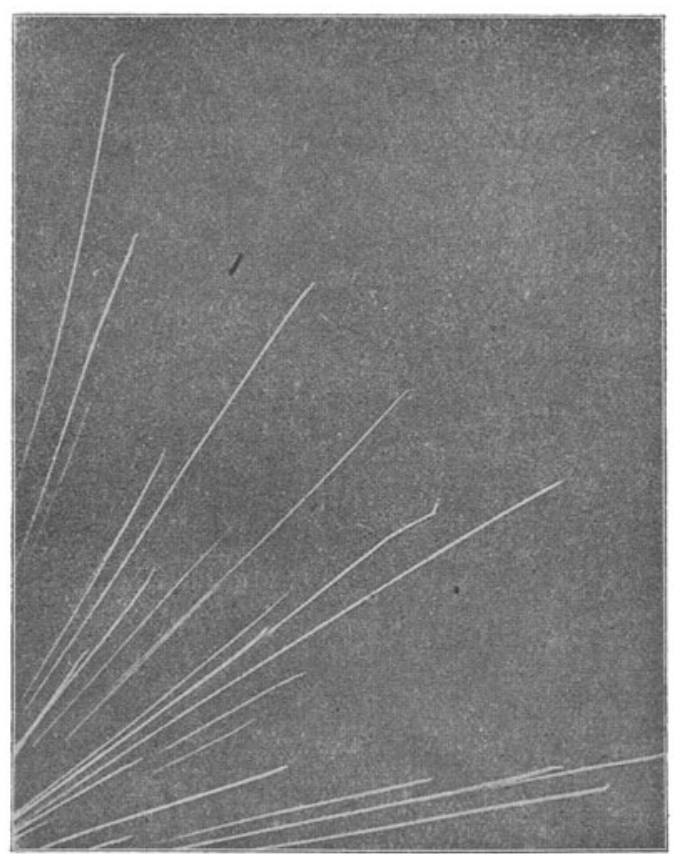

Fig. 2.

alle chemischen und physilialischen Eigenschaften des Atoms lestlegt. Das Atomgewisht läuft nur ungefähr der Ordnungszahl parallel, und daraus erklärt es sich, dab an verschiedenen Stellen des periodischen Systems (Ar-K, Co-Ni, Te-J) die Reihenfolge der Atomgewichte dem chemischen Verhalten nicht eatspricht. Der einwandfreie Beweis, daß die Kernladung der Atome von Stelle zu Stolle im periodischen System um eine Einheit zunimmt, wurde indegsen erst später mit Hilfe der Röntgenspektren (vgl. \& 14) erbracht. Nach unseren heutigen Kenntnissen besteht Wasserstofl aus einem einwertigen Kern $(x=1)$ und einem BJektron, Helium aus einem zweiwertigen Kern $(x=2)$ und zwei. Elektronen usw. bis zum Uran, welchem die Ordnungszahl $x=92$ entspricht. Im ganzen sind uns 
noch sechs Elemente (Ordnungszahlev: 43, 61, 72, $75,85,87)$ unbekannt.

Aus den Messungen über die Ablenkbarkeit der $\alpha$-Strahlen konnte Rutherford auch eine obere Grenze für die Dimensionen des Kernradius angeben, der sich (für Gold) zu 3. 10-12 em ergabi). Das ist im Verhältnis zur Gröbenordnung des Atoms $\left(10^{-8} \mathrm{~cm}\right)$ eine so kleine GröBe, daB man den. Kern für die Berechnung der ron ihm aus" gehenden Kraftfelder als punktfömig ansehen kann. Trotzdem zwingen uns die radiuaktiven Erscheinungen, dem Kern der schweren Elemente eine mehr oder weniger komplexe Strulktur zuzuschreiben: Bekannthich entsteht ein neues Wlement aus einer radioaktiven Muttersubstanz, entweder durch Aussendung eines o-Strahls (Heliumkern) oder eines $\beta$-Strahls (Elektrou). Der Chemismus der entstehenden Produkte wird von der FajansmSoddyschen Regel (1913) beheruscht, nach welcher bëi allen a-Umuandlungen wine Verschiebung zu der zweitniedrigeren Gruppe des periodischen Systems, bei allen B-Umwandlungen zur nächsthoheren Cruppe stattfindot. Vom eben dargelegten Standpunkt kann man das auch so aussprechen: Bei einex $\alpha$-Unwandlung nimmt die Kornladung um zwei Einheiten ab, bei einor $\beta$. Umwandlung um eine Einheit zu. Dicse Verhätnisse erkären sich zwanglos durch die Annahme, daß sowohl die -Strahlen (die zwei positive Elementarladungen tragen) wie die $\beta$-Strahlen (mit einer negativen) aus dem Kern stammen. Der Kern ist demnach wenigstens in radioaktiven Atomen ein komplexes Gebilde, zu dessen Bausteinen Elektronen und Heliumkerne gehören.

\section{Abschnitt.}

\section{Systeme mit einem Freiheitsgrad.}

\& 7. Anwendung der Wirkungsquanten auF das Rutherfordsche Atommodell. - Die Anwendung der Quantenlehre auf die Atomistik verdanken wir dem jungen dänischen Physiker Niels Bohr (1913). Seine Theorje verwertet in einel. äuBerst geschickten Art die in den $\$ \S 2-6$ besprochemen Elemente und trifft in so weitgehender Weise das Richtige, daß man sie als Markstein und Wendepunkt der ganzen Atomlehre bezeichnen kann. Der Planckschen Quantentheorie wurde durch sie ein neves, weites Anwendungsgebiet eroffnet, auf welchem wir trotz der Kürze der seither verflossenen Zeit schon über eine Reihe gesicherter theoretischer Kenntnisse verfügen.

Nach $\& 6$ besteht das Wasserstoffatom aus einem einwertigen Kern und einem Elektron. Wir wollen ein etwas allgemeineres Gebilde ins Auge fassen, nämlich einen Kern von der Ladung $t * e$, um welchen ein einziges Elektron kreist (Fig. 3a). Man nennt ein solches System, wasserstoffähnlich"; wenn $*$ von 1 verschieden ist, entspricht es nicht dem Normalzustand irgend

1) Aus weniger sicheren Foraussetzungen berechnet c. G. Danorin für Wasserstoff und Helium als obere Grenze des Radius 1,7.10-13 cm.

eines Atoms, denn um elektrisch neutral zu sein. fehlen ihm $\%$-1. Elektronen. Es ist also ein Atom. ron dem $x-1$ Elektronen abgetrennt sind, oder. wie man sagt, ein $(x-1)$ fach ionisiertes Atom. Wir wollen versuchen, in ähnlicher Weise, wie wir dies in $\$ \$ 2,3$ für den linearen Resonator getan haben, aus allen mechanisch möglichen Bewegungen des Elektrons nur gewisse quantentheoretisch zulässige herauszufinden. Dabei ergibt sich jedoch gegenüber dem dort betrachteten Fall ein wesentlicher Unterschied: In beiden Fällen geben die von einer elektrischen Ladung ausgefürten Schwingungen zi einer Ausstrahlung von Fnergie Veranlassung. Wahrend aber bei quasi-elastischer Bindung die Bewegung des Elektrons mit konstanter, vom Energieinhalt unablängiger Schwingungszabl erfolgt, ist dies bei Newtonschen Kräften nicht der Fall, der Energieverlust ändert vielmehr mit den Dimensionen der Bahn auch alle torigen Konstanten der Beweyung. Um diese Schwierigkeit hinwegzuräumen. sctzt sich Bohr in bewubten Gegensatz zur Elek rodynamik: cer nimmt einfach an, dap in den ruantenmäßig wusgezeichneten (,statischen"; Bahnen, auf die es uns schlieblich ankommt. Gerblung niwh statfindet. daB aber trotzdon:

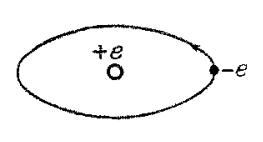

s.
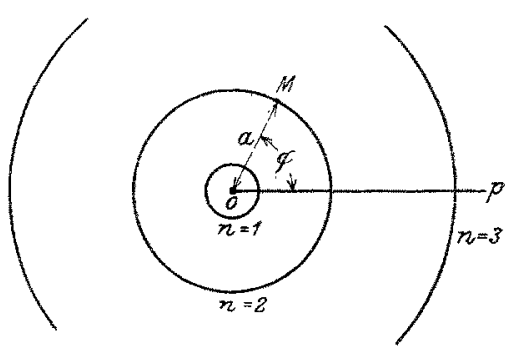

Fig. 4.

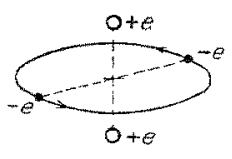$$
\text { . }
$$

die wechselseitige Anziehung ron Kern und Elektron nach den Gesetzen der Elektrostatik vor sicl geht. Die große Kühnheit dieser Annahme wird durch den glänzenden Erfolg gerechtfertigt.

Von diesem Standpunkt ist die Bewegung cines einzelnen Elektrons um einen Kern periodisch, und es ist nicht schwer, die Plancksche Bedingung (56) auf diesen Fall anzuwenden, wenn man ihn dadurch $z u$ einem System mit einem Freiheitsgrad macht, daß man nur kreisförmige Bahnen in Betracht zieht ${ }^{1}$ ). Die Lage $M$ des Planeten auf dem Kreise ist dann nämlich durch eine cinzige Koordinate bestimmt, als welche wir $z . B$. den Winkel $\varphi$ wählen können, den der Fahrstrahl zum Elektron mit einer festen Richtung o $p$ einschliebt (Fig. 4). Dabei überwiegt die

\footnotetext{
1) Bohr hatte bereits einen Vorläufer in Nicholson (1912).
} 
Masse des Kerms diejenige des Elektrons $(\boldsymbol{\mu})$ in solchem Maße, daß man mit guter Annäherung den Kern als uneudlich schwer ansehen und ihn durch ein festes Anziehungszentrum ersetzen kann. Mit dieser Aunäherung wollen wir uns in diesem und dem nächsten $\$ \S$ begnügen, und auf die Mitbewegung des Kerns erst in $\$ 10$ eingehen.

Es sei a der Radius der Kreisbahn, welche das Elektron um das feste Zentrum beschreibt und "die Geschwindigkeit in derselben, es sei ferner wie früher -e die (negative) Ladung des Elektrons, die positive des Kerns $x$ e. Bei Kreisbewegung mul die Zentrifugalkraft der Newtonschen Anziehung gerade die Wage halten, was zu folgender Beziehung zwischen Radius und Geschwindigkeit führt

$$
\frac{\mu v^{2}}{a}=\begin{gathered}
\gamma_{0} e^{2} \\
a^{2}
\end{gathered}, \text { oder } \mu v^{2}=\frac{z e^{2}}{a}
$$

Mit Hilfe dieser Gleichung erhält man für die Gesamtenergie $A$ des Elektrons, die sich aus kinetischer und potentieller Energie zusammensetzt

$$
A=\frac{1}{2} \mu v^{2}-\frac{* e^{2}}{a}=-\frac{x e^{2}}{2 a} \text {. }
$$

während sich für die Winkelgeschwindigkeit.

$$
\dot{\varphi}=\frac{v}{a}=\sqrt{\frac{\pi}{\mu a^{3}}} .
$$

ergibt.

Nach der gewöhnlichen Mechanik sind alle Werte des Radius a uad daher nach (12) auch (alle negativen) der Energie möglich. Wir wollen nun die Plancksche Quantenbedingung

$$
\int p d q=n h
$$

anwenden, um unter diesen Bahnen eine Auswahl zu treffen. Die Lagenkoordinate $(\alpha)$ ist hier der Winkel $\varphi$, und der ihm zugeordnete Impuls $p_{i f}$ ist nach den Regeln der Dynamik das sogenannte Winkelmoment der Bewegungsgröße $p_{\varphi}=\mu a v$, also eine konstante GröBe. Die Integration ist uber den ganzen Variabilitätsbereich der Veränlerlichen $\varphi^{\prime z u}$ erstrecken, also ron 0 bis $2 \pi$. Hiernach exhält man

$$
n h=\int_{0}^{2 \pi} p_{\varphi p} d \varphi=2 \pi p_{\varphi}=2 \pi \mu a \iota .
$$

Aus dieser Gleichung und der Beziehung (11) wewinnt man durch Eliminieren von $v$

$$
a_{n}=\frac{h^{2}}{4 \pi^{2} \gamma \mu e^{2}} n^{2}
$$

und das liefert in (12) und (13) eingesetzt

$$
A_{n}=-\frac{2 x^{2} x^{2} \mu}{h^{3}}-\frac{1}{n^{2}}, \quad \dot{\varphi_{n}}=\frac{8 x^{3} n^{2} \mu e^{4}}{h^{3}}-\frac{1}{n^{3}}
$$

Wir haben also aus allen mechanisch mög* lichen $a$ und $A$ eine Reihe diskreter quantenmäBig zulässiges Werte ausgewählt. D. h. das Elektron kann nicht in jeder beliebigen Entfernung rom Kern kreisen, sondern nur in einer der durch den Ausdruck (14) definierten statischen Bahnen. Wir sehen, daß mit, wachsender Quantenzahl n die Abstande benachbarter staticcher Bahnen im- mer gröber werden (die ersten Bahnen sind in Fig. 4 eingezeichnet). Umgekehrt ist es mit den statischen Energiestufen (15); diese liegen mit wachsendem $n$ immer dichter und häufen sich gegen den Wert $A=0$ (d. h. $a=\infty)$.

8. Die Bohrsche Frequenzbedingung. - Die Bewegung des Elektrons in einer statischen Babn, welche nach Obigem ohne Energieabgabe vor sich geht, bildet nach dem Gedanken von Bohr einen Normal- oder Gleichgewichtszustand des Atoms. Wird es durch irgend eine Störung aus einer solehen Bahn geworfen, so trachtet es, sofort auf einer andern ins Gleichgewicht zu kommen. Diese Endbahn muß natülich eine kleinere Energie haben als der Anfangszustand, da ein System ohne äußere Einflüsse nur Energie (durch Strahlung) abgeben, nicht aber gewinnen kann. Boht macht die Annahme, daß ein Atom 'nur während eines solchen Überganges des Elektrons von einer statischen Bahn auf eine andere zu strahlen vermag. und es entsteht die Frage, wodurch dabei die Wellenlänge der ausgesandten Strahlung bestimnt wird. Stellt man sich auf den in $\$ 4$ skizzierten Wien-Starkschen Standpunkt, so ist für die emitticrte Schwingungezahl die verfügbare Energie maßgebend. Wenn man also die Energien der Anfangs- und Endbahn mit $A_{m}$ und $A_{n}$ bezeichnet, erhält man in Analogie mit der Gleichung (6)

$$
c h v=A_{m}-A_{n} \text {..... (16 }
$$

Das ist in der Tat die zweite Hypothese, welche Bohr nebea der Planckschen Quantenbedingung in seiner Theorie benutzt ${ }^{\mathbf{1}}$ ). Wir wollen sie im Folgenden als, Bohrsche FrequenzJedingung" bezeichnen.

Man sieht, daß diese Hypothese auch dem Ritzschen Kombinationsprinzip $\$ 5$ gerecht wird und eine zwanglose physikalische Deutung desselben enthält. In der Tat stellt sich eine Schwingungszahl nach Formel (16) als Differenz zweier Termo dar, welche physikalisch die Energien zweier statischer Bahnen bedenten. Da der Ubergang eines Elektrons zwischen zwei beliebigen statischen Bahnen (in Richtung abnehmender Energie) nö̈glich sein soll, so kann man auch jeden Term mit jedem anderen. kombinieren.

\$ 9. Erklärung der einfachsten Seriengesetze. - Wenden wir uns wieder dem Falle des wases. stoffähnlichen Atoms zu, so brauchen wir nur den bereits gefundenen Ausdruck (15) für die Energie in (16) einzusetzen, um die Darstellung einer Anzah der in $\$ 5$ erwähnten Serien zu exhalten:

$$
v=\frac{2 \pi^{2} \gamma^{2} \mu e^{4}}{h^{3} c}-\left(\frac{1}{n^{2}}-\frac{1}{m^{2}}\right)=N x^{2}\left(\frac{1}{n^{2}}-\frac{1}{m^{2}}\right)
$$

Wenn $\operatorname{man} n=2$ setzt, stimmt dies der Form nach mit der Balmerschen Formel (7) überein.

1) Eis liegt uns fern, zu behaupten, daß Bohr die Arbeiten von Wien und stark wirklich gekannt und benutzt hat. Wir hielten es jedoch für zweckmäßig, in unserer Darstellung an bereits vorhandene Anschauungen anzuknüpfen. 
Aber auch numerisch erweist sich der Faktow $N$ mit der Rydbergschen Konstanten übereinstimroend, wenn man für die Konstanten $\mu, e, h$ die genauesten zurzeit aus anderen Erscheinuagen bekannten Werte einsetzt. Am besten verfährt man dabei in der Weise, dab man $N$ so schreibt:

$$
N=\frac{2 \pi^{2} \mu e^{4}}{h^{3} c}=\frac{2 \pi^{2}}{c} \cdot \frac{\mu}{e} \cdot\left(\begin{array}{l}
e \\
h
\end{array}\right)^{3}
$$

Die Verhältnisse $\frac{e}{\mu}\left(=5,2908 \cdot 10^{17}\right.$, Fortrat $1912)$ und $\frac{h}{e}\left(=1,370 \cdot 10^{-17}\right.$, Warburg und Milller 1915) lassen sich nämlich viel genauer bestimmen, als die Konstanten selbst. Das er* gibt für $N$ den numerischen Betrag

$$
N=110100 \text {, }
$$

der auf etwa 1,5\% genau sein müBte; wir sehen, dal ex wirklich mit dem experimentellen $(\$ 5)$ 109677,69 innerhalb der angegebenen Genauigkeitsgrenze übereinstimmt. Gegenwärtig verThhrt man umgekehrt und benutzt die optischen Daten zur schärfsten Bestimmung der Ciniversalkonstanten (vgl. \$ 13).

Der Kern des Wasserstoffatoms ist einwertig, daher hat man für dieses Gas in (17) $x=1 \mathrm{zu}$ setzen, und diese Formel ergibt für $n=1$, $n=2, n=3$ die drei Serien $(7 b),(7),(7 a)$ des Wasserstoffs. D. h. die Linien der ultraroten. Ritz-Paschenschen Serie werden beim Überspringen des Elektrons aus irgendeiner äußeren Bahn $(m=2,3,4 \ldots)$ in die erste, dem Kern nächste $(n=1)$ emittiert. Die Linien der Balmerserie entstehen während des Übergangs in die zueite statische Bahn, die der ultravioletten Lymanschen Serie während des Utberganges in die dritte.

Ein solcher Mechanismus der Entstehung der Linien mach仑 es verständlich, warum in Geililerröhren nux die 12 ersten Linien der Balmerserie zu beobachten sind: Für das Auftreten der zur Ordnungszahl $m$ gehörenden Linie ist es Vorbedingung, daß in einem Bruchteil der Atome das Elektron in der m-ten Bahn kreist. Es können sich aber offenbar um so leichter Bahnen von grobem Radius ausbilden, je weniger dicht das Gas ist, je seltener also die Sphäre eines Atoms von benachbarten Atomen und Molekülen gestört wird. Es ist daher anzunehmen, daß der Gasuruck in den Teilen der Fixsternatmosphïre. wolche 29 Linien aussenden, wesentlich kleiner ist. als der in GeiBlerröhren gebränchliche Druck.

\$10. Mitbewegung des Kerns. - Wix haben bereits in $\$ 7$ erwähnt, daB andere Atome als die des Wasserstoffs nur in ionisiertem Zustande die Voraussetzungen der Formeln (15) und (17) erfüllen: Z.'B. wärde sie für einfach ionisiertes Ielium, d. h. für ein Atom, das aus dem zweiwertigen Heliumkern $(x=2)$ und nur einem Elektron bestebt, währead das zweite abgetrennt ist, gelten. Man erhät in diesem Falle aus (17)

$$
v=4 N\left(\frac{1}{n^{2}}-\frac{1}{m^{2}}\right)
$$

was man auch so schreiben kann:

$$
v=N\left(\frac{1}{\left(\begin{array}{l}
n \\
2
\end{array}\right)^{2}}-\frac{1}{\left(\frac{m}{2}\right)^{2}}\right)
$$

Wir sehen, dab diese Formel die Serien (8a, b) des $\& 5$ in sich enthält, welche dent Wasserstoff zugeschrieben wurden. In der Tat. tür $n=3$ folgt der die Rydbergsehe (8a) und Fowlersche (8b) Serie umfassende Ausdruck:

$$
v=N\left(\frac{1}{1,5^{2}}-\frac{1}{\left(\frac{m}{2}\right)^{2}}\right), \quad n= \pm, 5,6, \ldots .19
$$

für $n=4$ eine Formel:

$$
v=X\left(\frac{1}{2^{2}}-\frac{1}{\left(\frac{m}{2}\right)^{2}}\right), \quad m=5,6,7, \ldots
$$

welche auBer den Linien der Pickeringserie ( $b$, für ganzzahlige $m$ eine Reihe anderer ergibt, welche mit den Wasserstofflinien der Balmerserie (7) zusammenfallen und deshalb. von Pickering nicht erwähnt wurden.

Dal die beobachteten Serien $(8,8 a, b)$ ihre Entstehung nicht Wasserstoff, wie man früher glaubte, sondern Helium verdanken, wie es die eben dargelegte Theorie fordert, hat sich vollauf bestätigt. Wir haben schon erwähnt, daß Fowles einen Zusatz von Helium zum Wasserstoff für die Erzeugung desselben unbedingt nötig fand; im Sommer 1914 konnten dann Paschen und Bartels diese Linien in reinem Helium beobachten. Aber schon Bohr selbst hat auf einen Umstand hingewiesen, der einen noch schlagenderen Beweis für die Richtigkeit seiner Auffassung beibringt: Wir wissen bereits, dab dic Linien der Fowlerschen Serie der Formel (8b) nicht ganz streng genügen, diese Abweichung erklärt sich vollständig, wenn man dio Rechnung etwas stren. ger durchführt und die Mitbewegung des Karns Jerücksichtigt.

In Wirklichkeit ist der Kern kein festes Zentrum, sondern hat eine endliche Masse $M$, weshalb Elektron und Kern Kreise um ihren gemeinsamen Schwerpunkt beschreiben. Die Radien der beiden Bahnen verhalten sich bekanntlich umgekehrt proportional den Massen $\mu$ und $M$. In die Quantenbedingung (11') ist nunmehr an Stelle vor $p_{\varphi}$ die Summe der Winkelmomente der Bewe. gungsgrößen von Kern und Elektron einzuführen, und man erhält nach einer analogen $Z$ wischen. rechnung für die Energie die gleiche Formel (17), in der jedoch die Gröbe $N$ nicht wehr durch den Ausdruek (18) gegeben ist, sondern auch von $M$ abhängt:

$$
\mathrm{V}=\frac{2 \pi^{2} \mu c^{4}}{h^{3} c} 1 \frac{1}{1+\frac{\mu}{M}} \ldots . .
$$

Bezeichnen wir den früheren Ausdruck, den wir erhielten, indem wir die Masse $M$ als unend- 
lich groß gegen $\mu$ ansahen, mit $N_{\propto}$, so schreibt sich:

$$
N=\frac{N_{\infty}}{1+\frac{\mu}{M}} . \cdots
$$

Demnach ist die Rydbergsche Zahl streng genommen keine aniverselle Konstante, sondern wechselt, wenn auch sehr schwach, von Element zu Element. Die größten Abweichungen von $N_{\infty}$ finden gerade für Wasserstoff und Helium statt, da für diese beiden Elemente das Verhältnis $\mu / M$ den gröbten numerischen Wert annimmt.

Die neuesten Messungen von Paschen (1916) ergaben die Rydbergsche Zahl

$N_{H}=109677,69, \quad N_{H e}=109722,14 \ldots(22$

Setzt man $e, h, M_{H}$ und $M_{H e}$ als bekannt voraus, so kann man mit Hilfe dieser Zahlen $e / \mu c$, $\mu / M_{H}$ and $\aleph_{\infty}$ berechnen. Man erhält.

$e / \mu c=1,76 \cdot 10^{7}, \quad M_{H} / \mu=1844, \quad N_{\infty}=109737,16$.

Der beste, direkt experimentell (aus Messungen am Zeemaneffekt) gefundene Wert für e/ $\mu c$ ist, wie bereits erwähat, $1,76,10^{7}$, die Ubereinstimmung also eine vollständige.

Dieser neue Triumph der Bohrschen Theorie war aut das Urteil der Fachgenossen von entscheidendem EinfluB. Frïher verhielt sich die Mehrzahl derselben reserviert; man gab allgemein zu, dab es Bohr gelungen war, die Rydbergsche Konistante aus den Universalgrößen $e$, $u, h$ aufzubauen, glaubte aber, daß sein Atommodell dabei eine mehr zufällige Rolle gespielt habe, und hielt dessen Leistungsfähigkeit mit diesen Ergebnissen für erschöpft. Daß man durch Steigerung der. Genauigkeit der Rechnungen neue wichtige Resultate erzielen kann, zeigte, daß es sich nicht nur um eine oberflächliche Analogie handelt, und regte verschiedene Physiker an, es in der Atomtheorie mit noch tiefer gehenden Anwendungen der Himmelsmechanik zu versuchen.

Kurz erwähnen wollen wir noch, wie nach der Meinung von Bohr das Wasserstoffmolekül gebaut sein muB. 'Dasselbe $\left(\mathrm{H}_{2}\right)$ besteht aus zwei Atomen, enthält also zwei positive Kerne uad zwei Elektronen. Die Anordnung dieser Bestandteile ist in Fig. $3 \mathrm{~b}$ wiedergegeben: Die beiden Elektronen bewegen sich in einern (in der Figur ausgezogenen) Kreis um die Verbindungslinie der beiden Kerne als Achse. Die quantentheoretisch zulässigen Dimensionen des Moleküls lassen sich in derselben Weise berechnen, wie die des Atoms (\$7), und man erhält für den Radius des innersten Kreises, den die Elektronen beschreiben können. und welcher dem Normalzustand des nicht leuchtonden Gases entspricht:

$$
\alpha^{\prime}=0,504 \cdot 10^{-8} \mathrm{~cm}, . . . .2\left(23^{1}\right.
$$

während Formel (14) für das Atom einen aur wenig verschiedenen numerischen Wert liefert:

$$
a=0,528 \cdot 10^{-8^{\circ}} \mathrm{cm} \text {. }
$$

Der halbe Abstand zwischen den beiden Kernen verhält sich zum Radius $a^{\prime}$ wie $1: \sqrt{3}$.

Der Wert (231) steht in guter Ưbereinstim- mung mit den Ergebnissen der kinetischen Gastheorie. Eine weitere Bestätigung des Bohrschen Molekelmodells verdanken wir Debye (1915), der die Dispersion eines aus solchen Gebilden. bestehenden Gases untersuchte und beim Vergleich mit den an Wasserstoff gemessenen Dispersionswerten eine volle Übereinstimmung fand.

III. Abschnitt.

Systeme mit mehreren Freiheitsgraden.

\$11. Erweiterung der Quantenbedingungen auf mehrere Freiheitsgrade. - Die Frage, wie die Plaacksche Bedingung (5) auf Systome mit mehreren Freiheitsgraden zu erweitern ist, wurde von Poincaré auf dem Brüsseler QuantenkongreB im Jahre 1911 aufgeworfen, aber erst vier Jahre später gleichzeitig von $M$. Planch und A. Bommerfeld bis zu einem gewissen Grade beantwortet. Während Planck dabei von allgemeinen statistischen Betrachtungen ausging, hatte Sommerfeld von vornherein die Anwendung auf das Bohrsche Atommodell im Auge, Da wir in den nächsten Paragraphen einige Spezialfragen besprechen wollen; für deren Behandlung sich die Sommerfeldschen Ansätze glänzend bewährt haben, schließen wir uns zunächst dieser Betrachtungsweise an. Auf Plancks nur formal von derselben verschiedene Theorie werden wir später $(\$ 16,17)$ zurückkommen.

Sommerfeld ging von der Tatsache aus, dab die Linien der Balmerserie nicht einfach sind, sondern bei Untersuchung mit Spektralapparaten von sehr starker Auflösung sich als mindestens doppelt erweisen. Da sich nach der Bohrsehen Theorie (\$ 8) eine Spektrallinie aus der Kombination zweier statischer Bahnen ergibt, schlol Sommerfeld, daf mehr statische Bahnen vorhanden sein müssen, als die Bohrsche Formel (17) angibt; und das veranlabte ihn, auch die Möglichkeit elliptischer Bahnen in Betracht zu ziehen.

Unter der Wirkung eines Newtonschen Anziehungszentrums beschreibt ein Körper im allgemeinen eine Ellipse (Keplerellipse), in deren Brennpunkt sich "das Zentrum befindet. Fs handelt sich also darum, unter allen möglichen elliptischen Bahnen, welche ein Elektron nach der Mechanik beschreiben kann, die quantenmäBig zulässigen oder statischen herauszufinden. Eine Ellipse wird nun nach Größe und Gestalt durch zwei Koustanten (etwa grobe und kleine Halbachse) bestimmt, und daher braucht man, um sie festzulegen, auch zwei Quantenbedingungen. Es sei die Lage des Elektrons in der Bahnebene durch. die Polaxkoordinaten $r, \varphi$ mit dem Kern (den wir wieder als festes Zentrum ansehen) als Ursprung bestimmt. Sommerfeld übernimmt nun die Quantenbedingung

$$
\int p_{\varphi} d \varphi=n h
$$

die sich bei Bohr bewährt hatte, und ergänzt sie durch die analoge Beziehung 


$$
\int p_{r} d r=n^{\prime} h, \ldots \ldots
$$

unter $p_{r}$ den dem Radiusvektor $r$ zugeordneten Impuls verstanden (,,Was dem $\varphi$ recht ist, ist dem $r$ billig!"). Beide Integrale sind über sämtliche Punkte der Bahn, also über eine Periode der Bewegung zu erstrecken.

Allgemeiner, wenn ein System durch $f$ Lagenkoordinaten $q_{1}, q_{2} \ldots, q_{\rho}$ und $f$ ihnen zugeordnete Impulse $p_{1}, p_{\mathbf{2}} \ldots p_{f}$ bestimmt wird, mul man nach Sommerfeld if Bedingungen von der Form $\perp$

$$
\int p_{i} d q_{i}=n_{i} h, \quad i=1,2,3, \ldots i \text {. }
$$

ansetzen. Da $p_{i}$ wnd $d q_{i}$ stets das gleiche Vopm zeichen haben, so folgt aus dieser Definition, dafi die $n_{i}$ stets positive ganze Zahlen sind.

Das Resujtat, welches die Bedingungen (24), (241) für die Halbachsen der statischen Keplerellipsen ergeben, besteht in Folgendem:

$$
a=\frac{h^{2}}{4 \pi^{2} m e^{2}}\left(n+n^{2}\right)^{2}, \quad b=a \frac{n}{n+n^{\prime}} .
$$

Bei gegebener Summe der Quantenzahlen $n+n^{\prime}$ ist also a konstant, $b$ veränderlich, und zwar ist das Verhältnis $b / a$ ein eehter Bruch mit
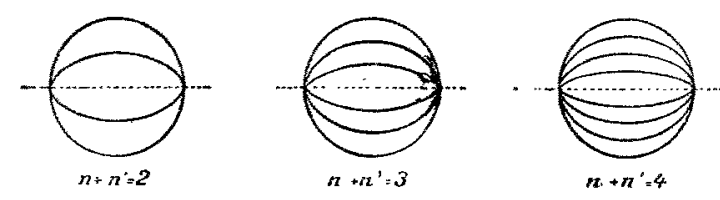

Fig. $5 a, b, c$.
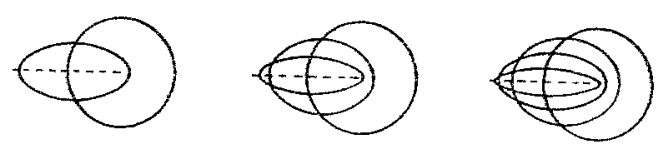

Fig. $6 a, b, c$.

dem Nenner $n+n$. Für die Werte der Quantensumme $n+n^{\prime}=2,3,4$ sind die möglichen Ellipsen in der Fig. 5 dargestellt. Dabei ist diejenige Bahn. welche in eine doppelt durchlaufene gerade Linie ausartet $(b=0, n=0)$, punktiert angedentet, denn in einer solchen Bahn müBte das Elektron mit dem Kern zusammenstoßen, und man kann sie daher als unmöglich ansehen. Deshalb ist die, Zahl der wirklich zustandelsommenden Bahnen in jedem Falle gleich $n+n^{\prime}$. Es ist zu bemerken, daß die Ellipsen zum Kern. in Wirklichkeit konfokal angeordnet sind (Fig. 6) und nicht konzentriscl, wie sie der Übersichtichkeit wegen in der Fjg. 5 eingezeichnet sind.

Der Zweck von Sommerfeld war also insofem erreicht, als er eine wesentlich größere $Z$ ahl von statischen Bahnen erhielt. Trotzden war das Restiltat zunächst eine Enttäuschung, denn die Vermehrung der Bahnen war von keiner Vermehrung der Energiestufen begleitet. Die Energie der Képlerellipse ist nämlich eine Funktion der sroken Aclıse allein, also für sämtliche Bahnèn einer jeden unserer Figuren $(5 a, b, c)$ die gleiche. Sie drückt sich durch die Formel aus:

$$
A=-\frac{2 \pi^{2} x^{2} \mu e^{1}}{h^{2}\left(n+h^{2}\right)^{2}},
$$

welche bei ganzzahligen $n$ und $n^{\prime}$ genau dieselber diskreten Werte ergibt, wie der Bohrsche Ausiruck $A=-2 \pi^{2} x^{2} \mu e^{4} / h^{2} n^{2}$.

Sommerfeld erhielt also dieselbe einfache Linienserie, die in Formel (17) enthalten ist, aber jede Linio entsteht bei ihm auf mehrfache Weise, aus mehreren verschiedenen Paaren statischer Bahnen. Sie enthält, sozusagen, mehrere zusammenfalleade Freiheitsgrade. Erst durch. Berücksichtigung der Veränderlichkeit der Masse des Elektrons als Funktion der Geschwindigkeit, wie sie die Relativitätstheorie fordert, ist es Sommerfeld gelungen, disse latenten Freiheitsgrade auseinander zu ziehen (\$ 13) und eine glänzende Übereinstimmung mit der Erfahrung zu erzielen.

\$12. Bedingt periodische Bewegungen. - In der Fassung der Quantenansätze, wie sie im letyten Paragraphen nach Sommerfeld gegeben wurde. blieben indessen noch einige Fragen offen. Sehon bei periodisehen Bewegungen, bei denen die Integration offenbar über eine Periode auszudehnen ist, war es nicht klar, welches von den vielen versehiedenen Koordinatensystemen, durch die man he Bewegung beschreiben kann, zu wählen ist. Bei nicht periodischen waren selbst die Grenzen der Integration unbekannt. Es bedeutete daher einen Fortschritt, als unabhängig $S c h$ warzschild und der Verfasser (1916), den Begriff der ,bedingt periodischen Bewegungen" aus der Himmelsmechanik in die Atomistik übernahmen und für diese erweiterte Blasse von mechanischen Systemen die Sommerfeldselien Quantenansätze nach Wahl der Koordinaten und Integrationsyrenzen präzisierten.

Als ,bedingt periadisch" bezeichnet man meehanische Systeme, zu deren Bestimmung man die Koordinaten so auswählen karn, daß, dieselben zwischen zwei festen Grenzen monoton hin- und herschwanken, oder, wie,man sagt, Librationen ausführen. Das einfachste Beispiel einer solchen Bewegung ist die ṫberlagerung von zueinander senkrechten Sinusschwingungen:

$$
x=x_{0} \sin \left(\omega_{x} t+\delta_{x}\right), \quad y=y_{0} \sin \left(\omega_{y} t+\delta_{y}\right)
$$

wo $x_{0}, y_{0}, \omega$ und $\delta$ Konstanten bedeuten. Man sieht sofort, dal $x$ im Laufe der Zeit $t$ immer die Werte ron $-x_{0}$ bis $+x_{0}$ (Librationsgrenzen) rorwärts und rückwärts durchläuft, ebenso $y$ diejenigen $z w i s c h e n-y_{0}$ und $+y_{0}$. Sind die Frequenzen $\omega_{x}$ und $\omega_{y}$ inkommensurabel, so kommt die Bahnkurve (Fig. 7) jedem Punkte des wwischen den Librationsgrenzen eingeschlossenen Rechtecks beliebig nahe, oder, um einen mathematischen Ausdruck zu gebrauchen, sie erfüllt das Rechteck überall dicht.

Ein anderes Beispiel bietet der für die Sommerfeldsche Theorie wichtige Fall der relativistischen Keplerellipse. Wr hahen hereits erwathnt, 
dab die Masse des Elektrons streng genommen nicht konstant ist, sondern von der Geschwindigkeit abhängt. Die Form dieser Abhängigkeit wird durch die Relativitätstheorie festgelegt. Berücksichtigt man diesen Umstand, so ergibt sich für die Bewegung unter dem Einfluß eines Newtonschen Kraftzentrums eine Änderung: Die Bahn ist zwar wieder elliptisch, jedoch steht die Ellipse nicht fest, sondern ihre groBe Achse rotiert mit einer kleinen Winkelgeschwindigkeit in der Bahnebene um den Brennpunkt mit demselben Umlaufssina, den das Elektron beim Beschreiben der Ellipse hat. Die Kurve, welche das Elektron dabei beschreibt, ist in Fig. 8 dargestellt. Hier variert wiederum die Koordinate $r$ zwischen den festen Librationsgrenzen $r_{1}$ und $r_{2}$, die ,zyklische" Variable $\varphi$ von 0 bis $2 \pi$. Das Ringgebiet $r_{1} \leqslant * \leqslant r_{2}$ wird im alluremeinen ron der Bahn ïherall dicht erfiullt.

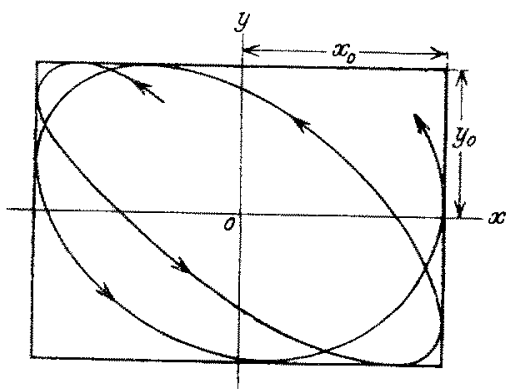

Fis.

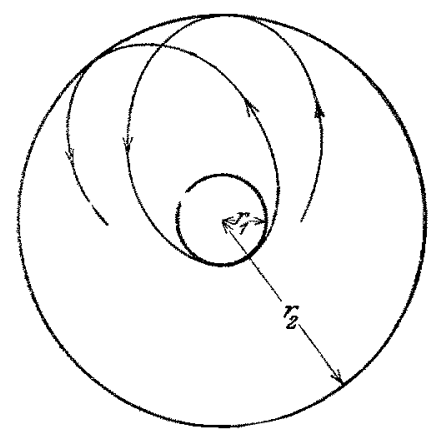

Eig. 8 .

Vom mathematischen Standpunkt besteht das Charakteristikum der bedingt periodisehen Bewesungen darin, dab, wenn man die Koordinaten in der eben angegebenen Weise wähltt), der einer jeden Koordinate $q_{i}$ zugeordnete Impuls $p_{i}$ nur von der Variablen $q_{i}$ allein, nicht aber von den ibrigen $q$ abhängs:

$$
p_{i}=p_{i}\left(q_{i}\right), \ldots \ldots .128
$$

wobei $p_{i}$ an deo Librationsgrenzen $\left(q_{i}=a_{i}, q_{i}=\right.$ $\left.b_{i}\right)$ verschwindet.

Der Quantenansatz für bedingt periodische Systeme in der rom Verfasser ausgesprochenen Form besteht nun daxin, daß man die Quanten-

1) Diese vahl der Koordinaten ist im allgemeinen eindeutig.

Nw. 1918 . integrale. (25) über einen Abschnitt der Bahn zwischen zwei aufeinanderfolgenden Berührungen ferselben Librationsgrenze erstreckt, oder, was dasselbe ist, zweimal von einer Librationsgrenze zur andern:

$$
{ }_{2}^{2} \int_{a_{i}}^{b_{i}} p_{i} d q_{i}=n_{i} h . . . . \cdot
$$

Ist die Koordinate zyklisch, wie der Winkel $\varphi$ im Falle der relativistischen Keplerellipse, so mul man von 0 bis $2 \pi$ integrieren:

$$
\int_{0}^{2 \pi} p_{z}^{-x} d q_{z}=n_{z} h
$$

Es läbt sich allgemein beweisen, daß diese Bedingungen, sofern ihnen sämtliche Freiheitsgrade unterworfen werden, die Energie als Funktion der Quantenzahlen n vollständig festlegen.

\$ 13. Feinstruktur der Wasserstofflinien. Als erste Anwendung dex für bedingt periodische Systeme aufgestellten Regeln wollen wir das bereits im letzten $\S$ erwähnte Beispiel der relativistischen Keplerellipse besprechen ${ }^{1}$ ). Dir Quantenbedingungen, lauten hier gemäß den in Fig. 8 versinnlichten Verhältnissen

$$
2 \int_{r_{1}}^{r_{2}} p_{r} d t^{2}=n^{r} h, \quad \int_{0}^{2 \pi} p_{q} d \varphi=n h .
$$

Durch Berücksichtigung der Relativität sind die Verhältnisse gegen diejenigen der in \$ 11 erÖrterten gewöbulichen Keplegellipse nur wenig rerschoben. Die Quantenbedingungen wählen also wieder aus allen mechanisch möglichen Bahven eine Reihe ,statischer" aus, welche mit guter f nnäherung durch die Ellipsen der Fig. 5 dargestellt werden. Zum Unterschied von jenem Fall sind jedoch die Energiestufen, welche zu den statischen Ellipsen derselben Figur gehören, nicht streng gleich, sondern um kleine Beträge voneinander verschieden. Der Näherungsausdruck fiì die Energie lautet nämlich ${ }^{2}$ :

$$
A=-\frac{N h c x^{2}}{\left(n+n^{2}\right)^{2}}-\frac{N h c x^{4} \alpha^{2}}{\left(n+n^{4}\right.}\left[\frac{1}{4}+\frac{n^{2}}{n}\right]
$$

Mit $\alpha$ ist hier eino Konstante bezeichnet, welche sich aus den Universalkonstanten $e, h$ und $c$ aif folgende Weise zusammensetzt

$$
\alpha=\frac{2 \pi e^{2}}{h a}
$$

Der physikalische Sinn dieser Zahl ist die (irr Verhältnis zur Lichtgeschwindigkeit gemessene) Geschwindigkeit, welche ein Elektron in dex ersten $(n=1)$ statischen Kreisbahn des Wasserstoff-

1) In Wirklichkeit wurde dieser Fall von Bommerfeld schon vor der Anfistellung der alloemeinen Quantenregeln (29). (29) für bedingt periodische Systeme bebandelt.

2) Es läßt sich auch der exakte Ausdruck für die Fnergie angeben, jedoch ist die Formel (31) für unseres Zwecke ibersichtlicher. 
atoms $(x=1)$ besitat. Der numerische Wert des uns interessierenden Quadrats ist

$$
\alpha^{2}=5,316 \cdot 10^{-5}
$$

und daher ist das relativistische Korrektionsglied stets klein gegen den ersten Term des Ausdrucks (31).

Die Wirkung dieses Korrektionsgliedes ist offenbar eine doppelte, denn erstens ergibt sich eine allgemeine Erhöhung des absoluten Betrages der Energie für alle Eilipsen mit derselben groBen Achse una den Wext

$$
\frac{N h c x^{4} a^{2}}{4\left(n+n^{\prime}\right)^{4}}
$$

zweitens erhöht sich die Energie der verschiedenen Ellipsen um verschiedene dem Achsenverhältuis $\frac{n^{\prime}}{n}$ proportionale Beträge.

$$
\frac{n^{\prime}}{n} \frac{N h c x^{4} \alpha^{2}}{\left(n+n^{\prime}\right)^{4}}
$$

Unter Heranziehung der Bohrschen Frequenzbedingung

$$
h c v=A_{1}-A_{2}
$$

erhalten wir demnach für die beim Übergang eines Elektrons aus einer statischen Bahn $\left(m, m^{\prime}\right)$ in eine andere $\left(n, n^{\prime}\right)$ ausgesandte Schwingungszahl

$$
\begin{aligned}
& v=N x^{2}\left[\frac{1}{\left(n+n^{\prime}\right)^{2}}-\frac{1}{\left(m+m^{\prime}\right)^{2}}\right]+\frac{N \alpha^{2} n^{4}}{4} \\
& {\left[\frac{1}{\left(n+n^{\prime}\right)^{4}}\left(1+\frac{4 n^{\prime}}{n}\right)-\frac{1}{\left(n+m^{\prime}\right)^{4}}\left(1+\frac{4 m^{\prime}}{m}\right)\right]}
\end{aligned}
$$

Bei kleinen Werten der Kernladung $\%$ unterscheidet sich dieser Ausdruck nur wenig fom ersten Glied, welches nach $\$ 9^{*}$ die Formel der Balmerschen oder einer ähnlichen Serie liefert. Die Schwingungszahlen (33) gruppieren sich deshalb bei vorgegebenen Werten dor Quantenzahlen eng um eine in der Formel (17) enthaltene Zahl und bilden die Feinstruktur einer wasserstoffählichen Linie.

Da es nach Fig 5 für ein vorgegebenes System von Zahlenwerten $\left(n, n^{\prime}, m, m^{\prime}\right) m+m^{\prime}$ mögliche Anfangsbahnen und $n+n^{\prime}$ Endbahnen gibt, so könnte eine wasserstoffähnliche Linie aus $\left(m+m^{\prime}\right) \cdot\left(n+n^{\prime}\right)$ Komponenten bestehen. Die Zahl der beobachteten Komponenten schien jedoch kleiner zu sein. Um eine Einschränkung herbeizufühen, lieb sich Sommerfeld durch den Gesichtspunkt leiten, daß die Quantenzahlen wesentlich positive GröBen sind; es liegt daher nahe anzunehmen, daB bei Bohrschen Übergängen nicht nur ihre Summe abnehmen mub, sondern dab sie anch einzeln nicht wachsen dürfen. $D$. h, es soll nicht nur die Ungleichung $m+m^{\prime}>n+n^{\prime}$ bestehen, sondern auch

$$
m \geqslant n, \quad m^{\prime} \geqslant n^{\prime} \text {. }
$$

Fassen wit zum Beispiel die Grundlinie der Balmerserie $\left(\boldsymbol{H}_{\alpha}\right)$

$$
v=N\left(\frac{1}{2^{2}}-\frac{1}{3^{2}}\right)
$$

ins Ange, so ist $m+m^{\prime}=3 . n+n^{\prime}=2$. Ws wären daher a priori $2.3=6$ Übergänge möglich, die jedoch durch die Ungleichungen (34) auf die 4 im folgenden Schema enthaltenen reduziert werden

$$
\begin{aligned}
& n=3, m^{\prime}=0 \\
& m=2, m^{\prime}=1 \\
& m=1, m^{\prime}=2
\end{aligned}\left\{\begin{array}{l}
n=2, n^{\prime}=0 \\
n=1, n^{\prime}=1
\end{array}\right.
$$

In der Folge hat sich indessen gezeigt, dal die Sommerfeldschen . Ungleichungen keine strenge, sondern nur eine angenäherte Gültigkeit haben: Auch die ihnen widersprechenden Linien treten unter Umständen (je nach der Erregungsart der Geillerohre) auf, jedoch immer mit nur schwachen Intensitäten.

Auch für die Inteusität der Komponenten gaibt die Theorie gewisse Anhaltspunkte. Statistische Betrachtungen machen es plawsibel, dab die Wahrscheinlichkeit ciner elliptisehen Bahn ihrem Achsenverhältnis $\left(\begin{array}{c}n^{\prime} \\ n+n^{\prime}\end{array}\right)$ proportional ist. Die Wah. scheinlichkeit eines Bohrschen Uberganges wäre dann proportional dem Produkt der entsprechenden Zahlen für die Anfangs- und Endbahn, also

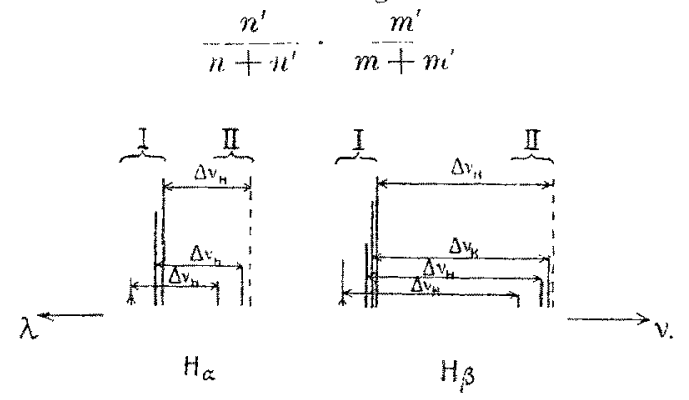

Fig. 1).

In der Tat folgt die Intensität der Komponenten ungefähr dieser Regel, wenn das betref. fende Gas durch Funkenentladung zum Leuchten gebracht wird, während die Verhältnisse bej Gleichstrom etwas anders zu liegen schëinen.

In Fig. 9 sind die Komponenten der beiden ersten Linien ( $H_{\alpha}$ und $H_{\beta}$ ) der Balmerserie $\left(n+n^{\prime}=2\right)$ nach 'Lage (in der Skala der Schwingungszahlen $v$ ) und Intensität eingezeichnet. Die irrealen Linien, d. h. diejenigen, welche den Quantenungleichungen (34) widersprechen, sind dabei punktiert oder durch einen kurzen Pfeil angedeutet, die theoretisch $z u$ erwartende Intensitat ist durch die Länge des betreffenden Striches yeranschaulicht. Charakteristisch tür die Anordnung der Linien ist das Auftreten konstanter Abstände zwischen verschiedenen Linienpaaren. Dies rührt daher, daß für die eine mögliche Endbahn (Fig. 5), etwa $n=2, n^{\prime}=0$, die Formel (33) $m+m^{\prime}$ mögJiche Werte von $v$, je nach Wahl der Anfangsbahn (Fig. 5) ergibt. Für die zweite Endbahn $(n=1$. $n^{\prime}=1$ ) erhält man $m+m^{\prime}$ weitere $v$. welche sich 
von denen der ersten Gruppe bezw. nur um die Differenz des von $n, n^{\prime}$ abhängigen Korrektionsterms in beiden Fällen, also um die konstante Differenz

$$
\Delta v_{H}=\frac{N \alpha^{2}}{16}
$$

unterscheiden. Die Größe $d v_{H}$ bezeichnet Sommerfeld als die Schwingungsdifferenz des Wasserstoffdubletts, ihr numerischer Wert is theoretisch nach den in $8 \$ 5$ und 13 angegebenen Werten von $N$ und $\alpha^{2}$

$$
A_{H}=0,364 \mathrm{~cm}^{-1} \cdot \cdot \cdot . \cdot(35 \mathrm{l}
$$

Beim Vergleich mit der Beobachtung ist indessen $z u$ berücksichtigen, daB die in Fig. 9 dargestellten Strukturen auch bei Benutzung dex stärksten optischen Mittel nicht vollständig aufgelöst werden können, sondern als einfache Dubletts erscheinen. Beim Wasserstoff sind nämlich die Komponenten etwas breit und verwaschen und die Abstände so eng, dab benachbarte. Linien leicht ineinanderfließen. Was man also als Du-

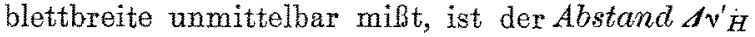
zwischen den Schwerpunkten der beiden engen Liniengruppen. Mit Benutzung der theoretisehen Intensitäten erhält man für $H_{\alpha} \quad(\lambda=6562,8)$

$$
A^{t} H=0,842 d v_{H}=0,307 \mathrm{~cm}^{-1} \text {, }
$$

oder in Wellenlängen umgerechnet:

$$
d \lambda_{H}=0,132 \text { A.-E. }
$$

\begin{tabular}{|c|c|c|}
\hline & $A \lambda^{\prime} H$ & $d v^{\prime} H$ \\
\hline Michelson ..... & 0,14 A.-E & $0,33 \mathrm{~cm}^{-1}$ \\
\hline Fabry und Buisson . . . . & $0,132 \quad "$ & 0,307 \\
\hline Meibner. . . . . . & 0,124 & 0,289 \\
\hline
\end{tabular}

Experimentell wurden die folgenden Werte ge: funden :

Die Ubereinstimmung mit dem theoretischen Wert ist eine glänzende.

Günstiger für eine Prüfung der Theorie liegen die Verhältnisse bei den wasserstoffähnlichen Serien (vergl. $\$ \$ 5,10$ ) des ionisierten Heliums $(\%=2)$. Denn erstens sind die Heliumlinien schärfer, zweitens ist infolge des Auftretens des Faktors $x^{*}$ im Korrektionsglied der Formel (33) die Feinstruktur der Heliumlinien im Vergleich zu den analogen Linien des Wasserstoffs um das Sechzehnfache vergrößert. Diese Messungen sind von Paschen (1916) mit aller in der Gegenwart erreichbaren Genauigkeit ausgeführt worden. Fig. 10 gibt das theoretische Bild und den experimentellen Befund bei zwei verschiedenen Erregungsarten für die Grundlinie der Fowlerschen Serie $(\lambda=4686)$

$$
v=4 N\left(\frac{1}{3^{2}}-\frac{1}{4^{2}}\right)
$$

Betrachten wir die in der Figur versinnlichten Ergebnisse, so sehen wir, daB dieselben den theoretischen Erwartungen in allen Einzelheiten entsprechen. Unvorhergesehene Komponenten sind nicht vorhanden; es fehlen nur die den Quantenungleichungen widersprechenden Linien IIIa, b bei Gleichstrom, und Id bei Funkenentladung; die Linie Ilc ist nicht gemessen worden, weil sich eine schwache Linie zwischen zwei starken auf der photographichen Platte nicht feststellen läBt; einzelne eng benachbarte Linienpaare fließen in der Beobachtung zusammen. Die Utbereinstimmung zwischen Theorie und Erfahrung in dieser Figur ist geradezu das Glanzkapitel, der gröBte Triumph der Quantentheorie.

Nicht weniger gut ist die Ubereinstimmung für die zweite Linje der Pickeringserie, wir wollen jedoch dieselbe übergehen und in Fig. 11 die Feinstruktur der dritten geben. Entsprechend den drei möglichen Endbahnen zerfallen hier, wie in Fig. 10, die Komponenten in drei Gruppen I, II, III, während jedoch dort die Gruppen I und II übereinandergreifen, sind sie hier vollständig ge-
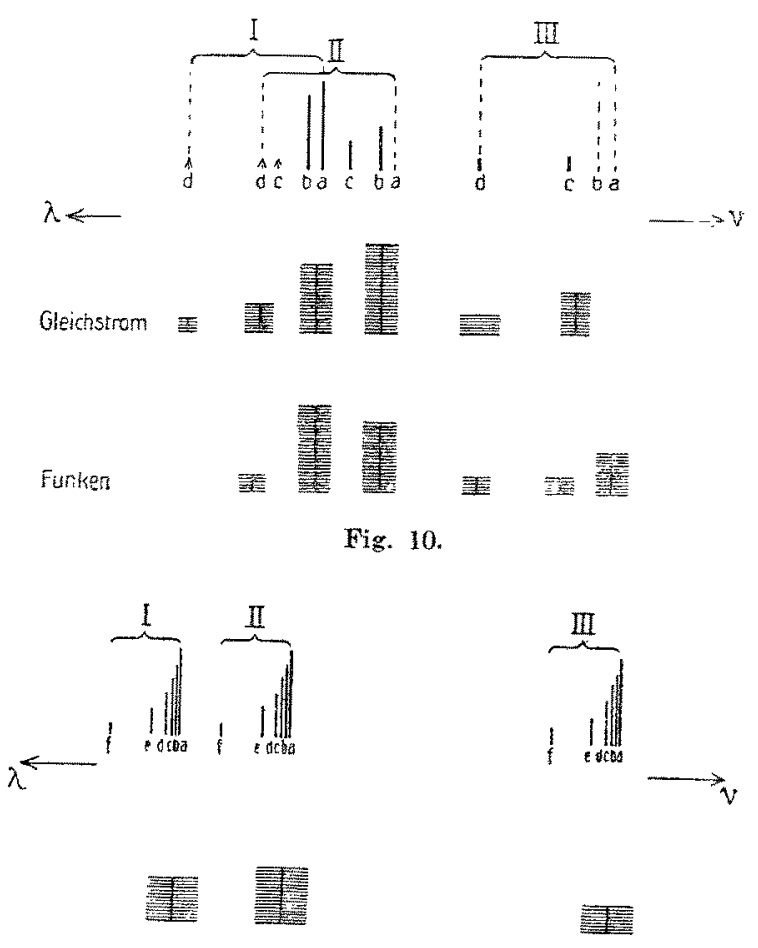

Fig. 11 .

trennt und so eng, dalj jede Gruppe in der Beobachtung als eine einzige breite Linie erscheint. Die $f$-Komponenten (welche übrigens teilweise den Ungleichungen (34) widersprechen), scheinen zu schwach fïr die Beobachtung zu sein.

Die Ausmessung aller dieser Feinstrukturen liefert als besten experimentellen Wert der Schwingungsdifferenz $\mathscr{A} v_{H}$

$$
A v_{H}=0,3645 \pm 0,0045
$$

Die Spektroskopie liefert uns somit drei GlöBen, welche nach der Theorie aus den Universalkonstanten ' $e, \mu, h$ aufgebaut sind: nämlich die Rydbergsche Zahl $N$ ( $\& 8$ ), die Änderung der Rydbergschen Zahl von Element zu Element infolge der Mitbewegung des Kerns (\$ 10) und die 
Schwingungsdifferenz des Wasserstoffdubletts $d v_{H}$. Aus den drei Gleichungen lassen sich die drei Unbekannten $e, \mu, h$ zahlenmäßjg bestimmen, und zwar dürfte diese Art dex Bestimmung bei der großen Genauigkeit der spektroskopischen Messungen schon gegenwärtig allen anderen Methoden ebenbürtig sein.

\$ 14. Spektra der Röntgenstrahlen. - Ưber the Emission und Absorption der Röntgenstrahlen ist in den Naturwissenschaften (Bd. 5, S. 513, 732) ein ausführlicher Bericht aus der Feder des IIm die experimentelle Erforschung dieses Gehiets hochverdienten $M$. Siegbahn erschienen, so tals iol diesen Gegenstand nur kurz zu streifen trinche.

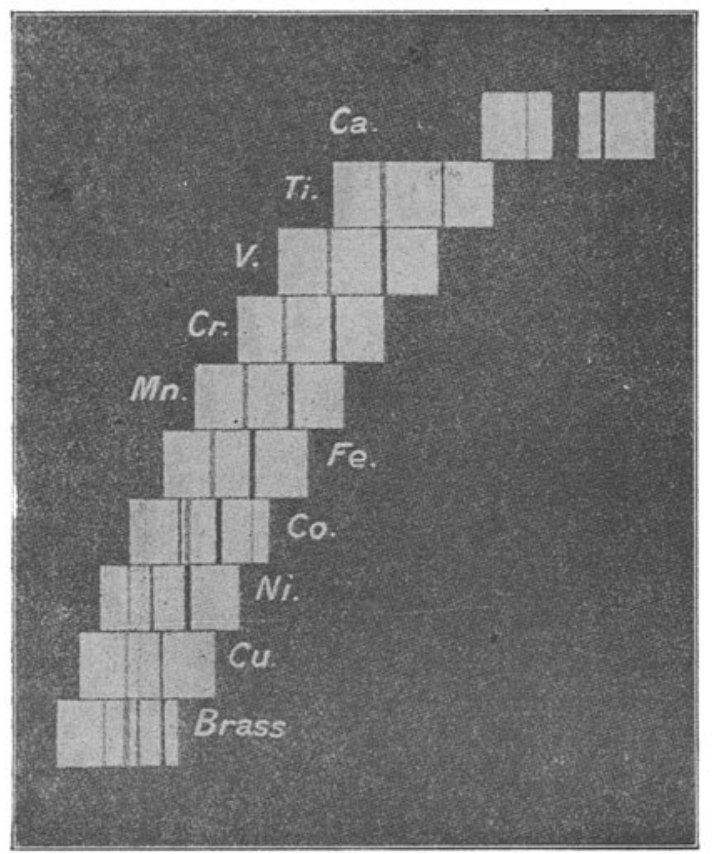

Fig. 1‥

Nachdem durch die Entdeckung von $v$. Laue, Wiedrich und Knipping die Möglichkeit einer - pektralanalyse im Gebiete der Röntgenstrahlen gegeben war, verdanken wir Moseley die ersten ausführlichen Untersuchungen über die Röntgenvellenlängen einer Reihe von Elementarstoffen. Die gemessenen Schwingungszahlen lassen sich in mehrere Serien einordnen, die man in der Reilhen. folge abnehmender Schwingungszahlen (Härten) als $K$-Sorie (Linien: $K_{:,} K_{3}, \ldots$ ), L-Serie usw. bezeichnet hat. In Fig. 12 ist eine von Moselen! tzemachte Aufnahme der Linien $K_{G}$ and $K_{3}$ alifinandexfolgender Elemente wiedergegeben.' Das unterste Bild der Reihe bezieht sich aul Messing (Brass), eine Legierung von Zink und Kupfer. Maß sich hiex die zwei $Z$ n-Linien einfach den heiden Cu-Linien der nächsthöheren Aufnahme therlagern, beweist, dal die Röntgenspektren eine rein additite Figenschaft des Atnms sind.
Die Lage del analogen Linien in dieser Figur zeigt einen parabolischen Verlauf. In der Tat konnte Moseley die Schwingungszahlen der $K e-$ Linie durch die Formel

$$
v_{K}=N(x-\cdots 1)^{2}\left[\begin{array}{cc}
1 & 1 \\
1^{2} & -2^{2}
\end{array}\right],
$$

diejenigen der $L_{\alpha}$-Linie durch

$$
v_{L}=V(x-i, 4)^{2}\left[\frac{1}{2^{2}}-\frac{1}{3^{2}}\right]
$$

darstellen, wobei $x$ die Wertigkeit des Kerns des untsprechenden Elementes bedeutet.

Dieses Ergebnis lehrt zweierlei: Erstens bestatigt sich die in $\$ 6$ dargelegte Auffassung, dalt lie Kernladung (im periodischen System) auteinanderfolgender Elemente um eine Einheit zurimmt. Zweitens sind die Röntgenlinien $K_{\text {" and }}$ $I_{\text {Lc }}$ wasserstoffähnlich mit der kleinen Modifikation, dab nicht, wie in der Balmerserie (7) bezt. (17), die ganze Kernladung $x$ wirksam, sondem ein Teil derselben, wie man sagt, ,abgesehirmt" ist.

Bei genauerer Betrachtung ergibt sich, dal liese Linien nicht einfach sind, sondern eina Feinstruktur besitzen. Theoretisch müBte man hei vollständiger Wasserstoffâhnlichkeit bei der Linie $L_{x}$ genau dieselbe Struktur wiẹ in dem durch Fig. 9 versinnlichten Fall der $H^{\mu}$-Linie des Wasserstoffs erwarten, mit dem Unterschied. das die Dimensionen derselben im Verhältnis $(x-7,4)^{4}$ vergrößert sind. In Wirklichkeit erhält man nur ein einfaches Dublett, dessen Schwingungsdifferenz allerdings zu domjenigen des Wasserstoffs im theoretisch richtigen Verhältnis

$$
A v=\left(x-7,4 i^{2} d v_{H}\right.
$$

sheht. Die Wasserstoffähnlichkeit ist also, wohl in Folge der komplizierteren Emissionsverhältnisse, eine beschränkte. Dagegen entspricht $K_{c}$ den Erwartungen der Theorie vollkommen und gibt, da hier die Endbahn einfach $\left(n+n^{\prime}=1\right.$ sder $n=1, n^{\prime}=0$ ), die Anfangsbahn doppelt ist, ein einfaches gegen $\Delta v_{H}$ im Verhältnis $(x-1)^{4}$ verbreitertes Dublett.

Die Messungen wurden vou Siegbahn und seinen Mitarbeitern für alle Elementarstoffe, soweit dies technisch möglich war, durchgeführt, und haben die Forderungen der Theorie bestätigt. Zur Illustration geben wir die nachstehende Fig. 13 wieder, welche das gesamte Beobachtungsmaterial der $K$-Serie zwischen Natrium $(x=11)$ und Neodym $(x=60)$ enthält. Als Ordinate ist $V v \cdot 10^{-4}$, als Abzisse $x$ aufgetraren. Der Verlauf der Quadratwurzel aus den Sohwingungszahlen ist in Übereinstimmung mit der Theorie ein linearer. $\alpha_{i}$ und $\alpha_{2}$ bedeuten dio beiden Komponenten der $K_{i z}$-Linie, $\beta_{1}$ und $\beta_{*}$ wind die Siegbahnsehen Bezeichnungen für $K_{\beta}$ und $K_{\gamma^{*}}$ Auch die Sehwingungsdifferenzen haben nach:genauen Messungen den won Sommerfeld rorausgesehenen Verlauf, und das bedeutet eino scharfe Probe der Theorie, wenn man bedenkt 
daß $\Delta v$ z. B. für $x=92$ (Uran, in der $L$-Serie gemessen) $(84,4)^{4}$, also rund 150 Millionen mal größer ist als für Wasserstoff. $\mathrm{Es}$ dürfte nicht riele. Theorien geben, die eine derartige Extrapolation auszuhalten vermögen.

Daß die Röntgenspektren teilweise wasserstoffähnlich sind, erklärt man sich auf die folgende Weise. Kreist ein Elektron in der Nähe des Kerns, zo üben die übrigen, welche sich in gröBerer Entfernumg bewegen, eine sehr geringe, zu vernachlässigende Rückwirkung auf dasselbe aus, und die Verhältnisse liegen im wesentlichen so, als
Nach einer Arbeit von Debye und noch unveroffentlichten Untersuchungen von $K r o o$ und Sommerfeld scheinen die Verhältnisse. so zu liegen: Das Atom besteht, wie es schon Bohr angenommen hatte, aus dem Kern und einer Reihe konzentrischer mit 'Elektronen besetzter Ring (Fig. 14). Den innersten bezeichnet man als $K$. Ring, den zweiten als $L$-Ring usw. Im Normalzustand haben diese Ringe eine ganz bestimmte Besetzung, z. B. haben wir in der Figur drei Elektronen im $K$-Ring and acht im $L$-Ring eingezeichnet. Die Vorbedingungen "für Röntgen-

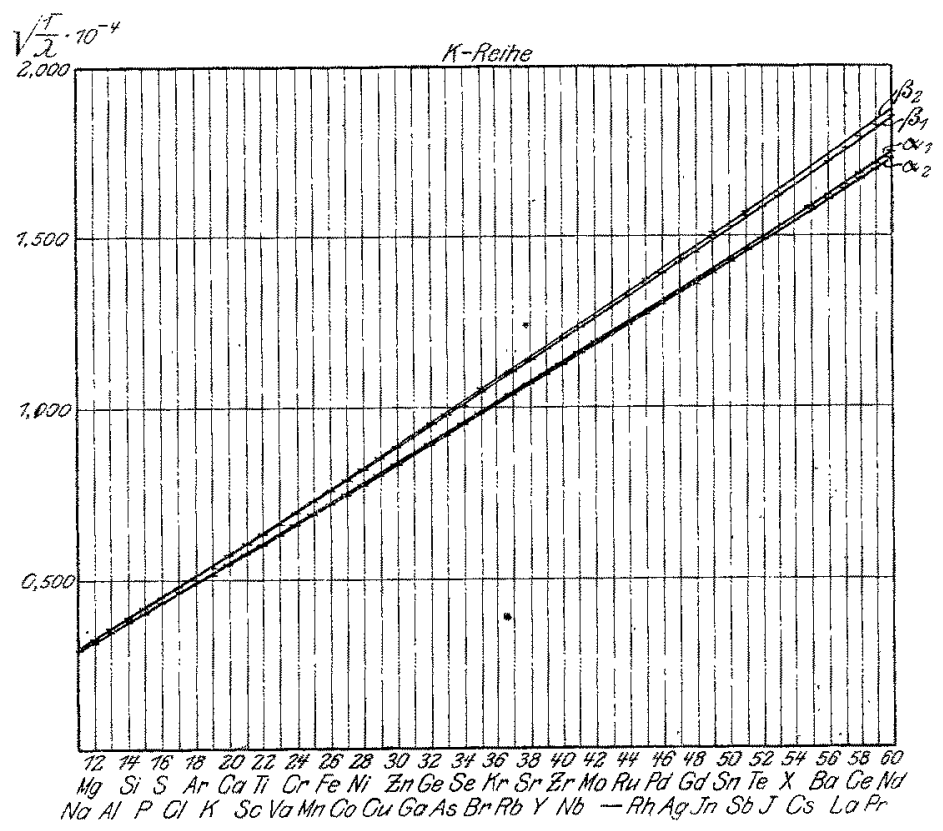

Fig. 13 .

ob nur ein $\%$-wertiger Kern and ein einziges Elektron vorhanden wären, wie in der Theorie der $\$ \S 7,8$ und 13 vorausgesetzt ist. Wie ist es aber zu deuten, dal ein Teil der Kernladung abgeschirmt erscheint? Eine mögliche Antwort auf diese Frage ist bereits bei Bohr gegeben: Stellen wir uns vor, daß nicht ein einzelnes. Elektron, sondern mehrere auf einem Kreisring angeordnete Elektronen um den Kern rotieren. Alsdann wirkt der Anziehung des Kerns eine Abstoßung zwischen den Elektronen entgegen, welche sich grerade darin äußert, daß an Stelle von $x^{2}$ ein kleinerer, Faktor $(x-\sigma)^{2}$ auftritt $\left.{ }^{1}\right)$ Daneben könnte man die Möglichkeit in Betracht ziehen, daß einige etwa $p$ Elektronen sich näher am Kern befinden als dasjenige, dessen Bewegumg wir studieren wollen; der Komplex aus einem *-wertigen positiven Kern und $p$ Elektronen wirkt dann auf größere Entfernungen ungefähr wie ein Kern mit $x-p$ positiven Ladungen.

1) Dabei kann jedes einzelne Elektron ev, auch eine elliptische Bahn beschreiben, aber die Elektronen eines Ringes müssen in jedem Augenblick die Eckpunkte eines regulatren Vielecks bilden.

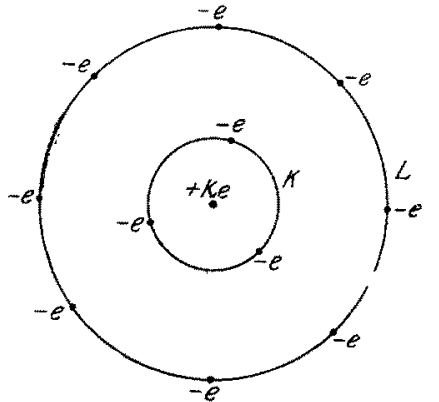

Fig. 14.

emission sind gegeben, wenn dureh die Wirkung eines äußerea Einflusses (Kathodenstrahls) ein Elektron aus einem inneren Ring in einen äußeren versetzt wird, z. B. aus dem $K$ Ring in den $L$-Ring. Dann hätte der erstere ein Elektron zu wenig, der letztere ein uberzähliges Elektron. Bei der Rückkehr des Elektrons in die Normallage auf dem innern Ring, welche unter Abgabe von Energie erfolgt, wird eine Röntgenwelle nach der Bohrschen Frequenzbedingung (16) ausgesandt. 
Die Durchführung dieser Vorstellungen ist noch nicht abgeschlossen und deshalb läBt sich noch nicht sagen, welches die definitiven Zahlen der Ringbesetzungen im Normalzustand sein werden. Vorläufig scheinen die Resultate für die Zahlen der Fig. 14, drei und acht Elektronen in den beiden innersten Ringen, zu sprechen; jedoch ist es keineswegs ausgeschlossen, dah sich die durch die Chemie (periodisches System) nahegelegten Werte $2,8,8,18,18$ usw. ${ }^{1}$ ) bestätigen werden.

\$15. Theorie des Starkeffelkts. - Bringt man win strablendes Atom in ein elektrisches Feld, so wird der Charakter der Emission durch das. letztere verändert: Spektrallinien, die ohne Feld einfrch erscheinen, werden in vielen Fällen durch die elektrische Kraft in mehrere Komponenten aufgespalten. Diese überraschende Entdeckung rerdanken wir Johannes Stark (1913), weshalb der neue Effekt seinen Namen erhielt. Obwohl die Wirkung mittlerer Feldstärken keineswegs sehwach ist, und zu ihrer: Beobachtung keine vxtrem feinen optischen Hilfsmittel benutzt zu werden brauchen, lagen die Verhältnisse für eine zufällige Entdeckung der Erscheinung insofern sehr ungünstig, als es äußerst schwer ist, in einem leuchtenden Medium auch ein mittelstarkes elektriches Spannungsgefälle aufrecht zu erhalten. Erst Stark, der nach diesem Effekt systematisch suchte, gelang es, die erwähnte Schwierigkeit durch sine sinnreiche Anordnung zu überwinden: Er brachte die verdünnte Gasschicht zwischen den Belegungen eines in einer Vakuumröhre befindlichen Kondensators durch hineintretende Kanalstrahlen zum Lenchten.

Auf diese Weise Lonnte er eine ganze Reihe ron Substanzen untersuchen, für unsere Zwecke sind aber die sehr sorgfältigen Messungen, die er über die ,elektrische Feinzerlegung“ der vier: ersten Linien der Balmerserie des Wasserstoffs $\left(H_{\epsilon}, H_{\beta}, H_{Y}, H_{j}\right)$ ausführte, von besonderer Wichtigkeit. Es zeigte sich, daß die Komponenten, in welche diese Linien aufgespalten werden, symmetrisch um die Normallage angeordnet sind, und dab die Aufspaltung proportional zur elelitrischen Feldstärke mit derselben anwächst. Es wurde senkrecht zur Feldrichtung beobachtet, und bei dieser Anordnung erwiesen sich die Komponenten als linear polarisiert.

In den Tabellen I bis IV sind weiter unten die Starkschen Resultate unter der Uberschrift .gemessen" zusammengestellt. Mit $p$ sind die Komponenten bezeichnet, deren elektrischer Vektor parallel zur Feldrichtung schwingt, mit $s$ diejenigen, bei welchen er senkreeht zur Feldrichtung orientiert ist. Unter $\Delta \lambda$ ist der Abstand der betreffenden Komponente (und der zu ihr symmetrischen) in Angströmschen Einheiten ein-

1) Vgl. J. J. Thomson, Korpuskulartheorie der Materie, 6. Kap. Brannsehweig 1908; N. Bohr, Pbil. Mag. 26, S. 8577, 1913; W. Kossel, Ann. d. Phys. 49, s. 229, 1916. getragen, unter "Int." ihre relative Intensität, (fr. bedeutet ,fraglich"), und zwar sind die Intensitäten nur innerhalb einer Spaite vergleichbar, und nicht von Spalte zu Spalte oder von Tabelle zu Tabelle. Die elektrische Feldstärke $E$, zu welcher die tabellierten Werte von $\Delta \lambda$ gehören, ist mit einiger Unsicherheit behaftet und wird von Stark auf 1.04000 Volt/cm geschätzt. Eine

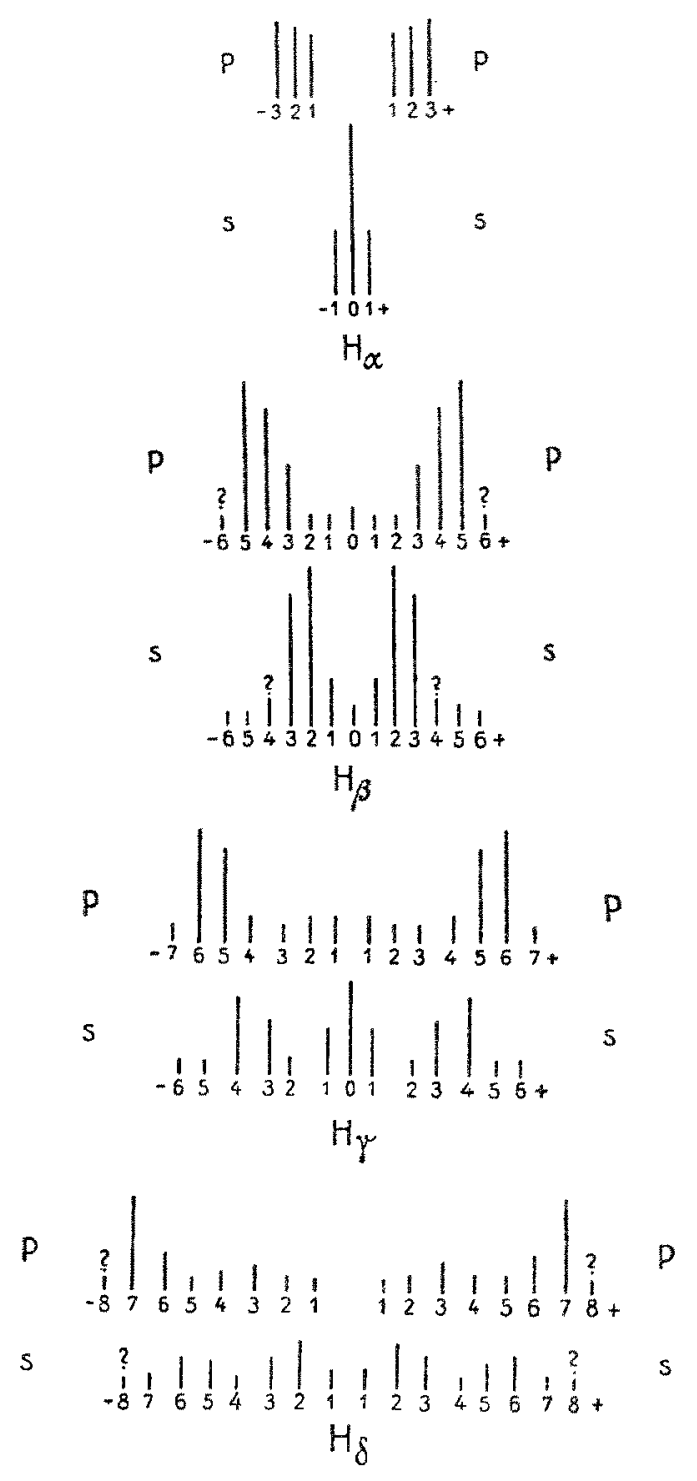

Fig. 15.

graphisehe Veranschaulichung des Aufspaltungsbildes bietet Fig. 15, in der die Intensität der Komponenten durch die Länge der entsprechenden Striche angedeutet ist, und diese, von der Normallage (0) ausgehend, der Reihenfolge rach nummeriert sind.

Die Theorie des Starkeffekts der Balmerserie reduziert sich auf die Betrachtung der Bewegung eines wasserstoffähnlichen Atoms $(\xi \tau)$ in einem 
homogenen elektrischen Felde. Diese Bewegung ist bei Vernachlässigung der Relativitätskorrektion eine bedingt periodische, und zwar sind es parabolische Kooratinaten, welche die Darstellung der Impulse in der durch Gleichung (28) gegebenen Form bewirken ${ }^{1}$ ). Die Koordinatenflächen entstehen durch Rotation der Fig. 16 um die Gerade $\xi=0, \eta=0$ als Achse. Es sind also zwei Scharen von konfokalen Rotationsparaboloiden ( $\xi=$ const, und $\eta=$ const) und die Meri-

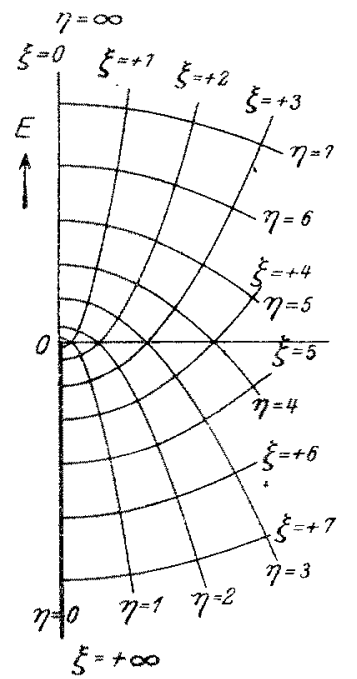

Fig. 16 .

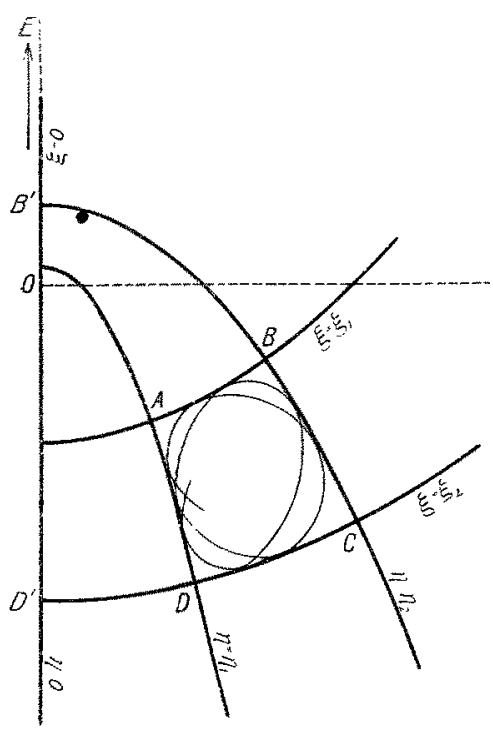

Fig. 17 .

dianebenen ( $\varphi=$ const, wenn man durch $\varphi$ den Azimut oder Winkel, welchen die rotierende Ebene mit einer festen Lage einschlieBt, bezeichnet). Ein Punkt des Raumes ist demnach durch die Koordinaten $\xi ; \eta, \xi$. festgelegt.

1) P. S. Epstein, Ann. d. Phys. 50, S. 489, 1916.
Wir wollen von der Mitbewegung des Kervs bsehen und ihn als festes Zentrum auffassen. Dann ist das Koordinatensystem so $\mathrm{zu}$ orienticren, daB der Kern mit dem Nullpunkt zusammenfallt und die Gerade $\xi=0$ die Richtung des elektrischen Feldes hat. Die Auflösung des Problems zeigt, daß die Bewegung des Elektrons in einem ringförmigen Raum eingeschlossen ist, welcher durch Drehung des krummlinigen Vierecks $A B C D$ (Fig. 17) um die Achse $\xi=0, \eta=0$ entsteht. Cud zwar muB man sich das so vorstellen, dab das Elektron die ins Viereck eingezeichnete Kurve durchläuft, während gleichzeitig die Figur (mit variabler Winkelgesehwindigkeit) um die erwähnte Achse rotiert.

Die Lage der Librationsgrenzen (vgl. \& 12) $\xi=\xi_{1}, \xi=\xi_{2}, \eta=\eta_{1}, \eta=\eta_{2}$, hängt natürlich von der Anfangslage und Anfangsgeschwindigkeit des Elektrons $a b$ und ist für jeden individuellen Fall der Bewegung eine andere, so daß durch geeignete Wahl der Anfangsbedingungen den GröBen $\xi_{1}, \xi_{2}$, $\eta_{1}, \eta_{2}$ jeder beliebige Wert erteilt werden kann. Im Interesse des $\$ 17$ wollen wir drei Grenzfalle ins Auge fassen. Zunächst lassen wir $\eta_{1}$ mit $\eta_{2}$ und $\xi_{1}$ mit $\xi_{2}$ zusammenfallen; dann zieht sich das Viereck auf einen einzigen Punkt zusammen. und das Elektron bewegt sich in einer zur Feldstärke senkrecht stehenden Kreisbahn. Andererseits können wir $\xi_{1}=0$ und $\eta_{1}=0$ setzen, die Bahnkurve erfullt dann das durch Rotation des Dreiecks $B^{\prime} C D^{\prime}$ entstehende Gebiet. Lassen wir nun auch noch $\xi_{2}$ immer kleiner uad schlieBlich gleich Null werden, so wird das Elektron auf die Bewegung in einer geradlinigen Strecke beschränkt: Es pendelt im Halbstrahl $\xi=0 \mathrm{zwi}-$ schen den Punkten 0 und $B^{\prime}$ hin und her, washalb ich diese Bahn als Pendelbahn bezeichnet habe. Den dritten ausgearteten Fall erhalten wir, wenn wir aicht $\xi_{2}$, sondern $\eta_{2}$ zu Null abnehmen lassen; es ist die ganz analoge Pendelbahn im Halbstrahl $\eta=0$ zwischen den Punkten 0 und $D^{\prime}$. Im Falle der Keplerellipse (ohne elektrisches Feld, \$11) haben wix den Fall der geradlinigen Bahn als wnwahrscheinlich verworfen und dies Vorgehen durch den experimentellen Befund der Feinstrukturen gerechtfertigt gefunden. Die Pendelbahnen scheinen a priori ebenso unwahrscheinlich; merkwürdigerweise treten aber die $z u$ ihnen gehörigen Komponenten im Starkeffekt auf, wenn auch durchweg mit äuBerst geringrer Intensität.

Im allgemeinen, nicht ausgearteten Fall der Bewegung haben wir nach (29), (29) die Quantenbedingungen in folgender Form zu sehreiben

$$
\begin{gathered}
2 \int_{\xi=}^{\varepsilon_{2}} p_{\xi}(\xi) d \xi=n_{1} h, \quad 2 \int_{\eta_{1}}^{\eta_{2}} p_{\eta}(\eta) d \eta=n_{2} h, \\
\int_{\hat{0}}^{2 \pi} p_{\varphi} d \varphi=n_{3} h,
\end{gathered}
$$


nnd aus diesen drei Beziehungen ergibt sich die Energie mit guter Näherung zu

$$
\begin{gathered}
A=-\frac{N x^{2} h c}{\left(n_{1}+n_{2}+n_{3}\right)^{2}} \\
+\frac{3 h^{2} E}{8 \pi^{2} \times \mu e}\left(n_{1}+n_{2}+n_{3}\right)\left(n_{1}-n_{2}\right) .
\end{gathered}
$$

Wie die Relativitätskorrektion, hat also auch ein elektrisches Feld die Wirkung, daß es dic Zahl der Energiestufen vermehrt und daher; sozusagen, die in einer Spektrallinie vorhandenen, aber zusammenfallenden Freiheitsgrade (Fntstehungsmöglichkeiten) auseinanderzieht $n$ nd sichtbar macht.

Nach der Bohrschen Frequenzbedingung (16) erlält man für die Schwingungszahl gegenüber der Normallage (17) eine Verschiebung vom Betrag

$$
\Delta v=-\begin{gathered}
3 h \\
8 \pi^{2} x \mu t c \\
Z=\left(m_{1}+m_{2}+m_{3}\right)\left(m_{1}-n_{2}\right) \\
\cdots\left(n_{1}+n_{2}+n_{3}\right)\left(n_{1}-n_{3}\right)
\end{gathered} \mid \cdots(3
$$

Wie frühex beziehe山 sich die Quantenzallen $m$ und $n$ auf Anfangs- und Endbahn, für welche bei Komponenten einer und derselben Linie resp. $m_{1}+m_{2}+m_{3}=$ const. and $n_{1}+n_{2}+n_{3}=$ const. gilt.

Aus der Struktur dieses Ausdrucks ersehen wir sofort zwei wichtige Eigenschaften des Starkeffekts wasserstoffähnlicher Linien: Erstens geht die Aufspaltung proportional mit der Feldstärlce $E$, zweitens ist sie symmetrisch. In der Tat, vertauschen wir die numerischen Werte von $m_{1}$ und $m_{2}$ und gleichzeitig die von $n_{1}$ und $n_{2}$, so wechselt $Z$ das Vorzeichen; d. h. zu jedem positiven $\Delta v$ gibt es ein gleich großes negatives. Wir haben bereits örwähnt, daß Stark wirklich ein solches Verhalten der Wasserstofflinien festgestellt hatte.

Der numerische Wert des Koeffizienten ist für Wasserstoff $(x=1) 6,43 \cdot 10^{-5}$, wenn $E$ in volt $/ \mathrm{cm}$ angegeben wird, und deshalb kang man für die Verschiebung einer Komponente in der Skala der Wellenlängen $\lambda$ schreiben

$$
\boldsymbol{A} \lambda=\lambda^{2} \boldsymbol{A} \mathrm{v}=6,43 \cdot 10^{-5} \lambda^{2} E \cdot Z \mathrm{~cm} . \quad .(38
$$

Die Auswahl der möglichen Werte von $Z$ wird durch die Sommerfeldsehen Ungleichungen (3t) eingeschränkt, welche in diesem Falle lauten

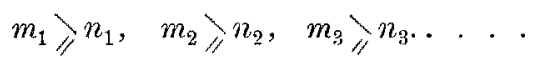

Die Werte von $\Delta \lambda$, welche die Reclnnung ergab, sind in den nachstehenden Tabellen zusam-

\begin{tabular}{|c|c|c|c|c|c|c|c|c|}
\hline & \multicolumn{4}{|c|}{$m_{1}+m_{2}+m_{8}=3$} & \multicolumn{4}{|c|}{ 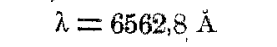 } \\
\hline & \multicolumn{4}{|c|}{ Berechnet } & \multicolumn{4}{|c|}{ Gemessen } \\
\hline 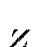 & \multicolumn{2}{|c|}{$m_{3}-n_{3}=2$} & \multicolumn{2}{|c|}{$m_{3}-n_{3}=2 l+1$} & \multicolumn{2}{|c|}{ p.Komp. } & \multicolumn{2}{|c|}{$s$-Komp. } \\
\hline & $\Delta \lambda$ & $Q_{m}$ & $1 \lambda$ & $Q_{m}$ & $J \lambda$ & Int. & $d \lambda$ & Int. \\
\hline$\tilde{0}$ & 14,7 & * & & & - & & & \\
\hline 4 & 11,7 & 1 & & & 11,5 & 1,2 & & \\
\hline$\because$ & 8,8 & 1 & & & 8,8 & 1,1 & & \\
\hline 2 & 5,9 & 1 & 5,9 & $*$ & 6,2 & 1 & - & \\
\hline 1 & 2,9 & * & 2,9 & (1) & - & & 2,6 & 1 \\
\hline 0 & - & & & $(1)$ & & & 0 & 2.6 \\
\hline
\end{tabular}
mengestellt. Dabei wurde als Feldstärke für die Linien $H_{a}$ und $H_{\beta} 106000$, für $H_{\gamma} 109000$ und

\begin{tabular}{|c|c|c|c|c|c|c|c|c|}
\hline & \multicolumn{4}{|c|}{$m_{1}+m_{2}+m_{3}=4$} & \multicolumn{4}{|c|}{$\Lambda=4861,3,3$} \\
\hline & \multicolumn{4}{|c|}{ Berechnet } & \multicolumn{4}{|c|}{ Gemessen } \\
\hline \multirow{2}{*}{$Z$} & \multicolumn{4}{|c|}{$m_{3}-n_{3}=2 l m_{3}-n_{3}=2 l+1$} & \multicolumn{2}{|c|}{$p-$ Komp. } & \multicolumn{2}{|c|}{ s. $\mathrm{Komp}$. } \\
\hline & $A \lambda$ & $Q_{m}$ & $d \lambda$ & $\Omega_{m u}$ & $\Delta \lambda$ & Iut. & $\Delta \lambda$ & Int. \\
\hline 12 & 19,4 & $*$ & {$[19,4]$} & 3 & 19,4 & 1 & 19,3 & 1 \\
\hline 10 & 16,1 & 2 & {$[16,1]$} & 3 & 16,3 & 11,5 & 16.4 & 1.1 \\
\hline 8 & 12,9 & 2 & 12,9 & 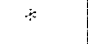 & 13,2 & 9,1 & 13,2 & 1.3 \\
\hline 6 & 9,7 & 2 & 9.7 & 1 & 10.0 & 4.8 & 9.7 & 9.7 \\
\hline 委 & 6,5 & $*$ & 6,5 & 1 & $6 . \overline{3}$ & 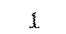 & 6.6 & 12,6 \\
\hline 2 & 3,2 & (2) & 3.2 & 1 & 3,3 & 1,2 & 8,4 & 2,3 \\
\hline 0 & 0 & (2) & $u$ & 1 & 0 & 1.4 & 0 & 1.4 \\
\hline
\end{tabular}
für $H_{\text {; }} 110000$ volt $/ \mathrm{cm}$ angenommen.
Tabelle I (Ha-Linie).

\begin{tabular}{|c|c|c|c|c|c|c|c|c|}
\hline & & $-m_{3}$ & $m_{3}=$ & & & -4 & $\omega, 0$ & \\
\hline & & Bere & net & & & Gem & sssen & \\
\hline 7 & $m_{3}-$ & $3=2$ & $m_{3}-n$ & $=2 l+1$ & $p-\mathrm{K}$ & omp. & $s-K_{c}$ & $\mathrm{mp}$ \\
\hline & $J \lambda$ & $Q_{m}$ & $\Delta \lambda$ & $Q_{m}$ & $d i$ & Int. & $\Delta \lambda$ & Int. \\
\hline 21 & 28,0 & $\%$ & 一 & & 29,4 & $1(\mathrm{fr})$. & & \\
\hline 20 & -- & & {$[26,6]$} & 4 & & & 26,3 & 1 \\
\hline 18 & 23,9 & 3 & - & & 29,9 & 0,8 & & \\
\hline 17 & - & & {$[22,7]$} & 4 & & & 22.8 & 1,1 \\
\hline 16 & - & & 21.3 & $\%$ & & & - & \\
\hline 15 & 20,0 & $: 3$ & - & & 19,9 & 7,2 & & \\
\hline 13 & - & & 17,3 & 2 & & & 17,3 & 6,1 \\
\hline 12 & 16,0 & 3 & - & & 15.9 & 2,0 & & \\
\hline 11 & 14,4 & $\%$ & - & & - & & & \\
\hline 10 & - & & 13,3 & 2 & & & 13,8 & 4,3 \\
\hline 9 & 12,0 & $*$ & - & & - & & & \\
\hline 8 & 10,6 & 2 & - & & 10,6 & 1 & & \\
\hline 7 & - & & 9,3 & 2 & & & 9,7 & 1,2 \\
\hline 6 & - & & 8,0 & x & & & - & \\
\hline$\tilde{0}$ & 6,7 & 2 & - & & 6,6 & 1.5 & & \\
\hline 4 & - & & 5,3 & $\%$ & & & - & \\
\hline 3 & - & & 4,0 & (3) & & & 3,9 & 3,6 \\
\hline 2 & 2,7 & 2 & - & & 2,6 & 1,6 & & \\
\hline 1 & 1,3 & $\%$ & - & & - & & & \\
\hline 0 & - & & 0 & (3) & & & 0 & 7,2 \\
\hline
\end{tabular}

Tabelle Il (IIb-Linie).

Tabelle III (H, - Linie).

Man sieht, daß die Übereinstimmung zwischen Rechnung und Erfahrung eine vorzügliche ist. Die stärkeren Komponenten Iiegen genau an den berechneten Stellen, nur bei sehr schwachen Linien, deren Lage schwer zu messen ist, sind die Abwreichungen mitunter etwas größer. Mit einem 
Tabelle IV (Hס - Linie).

$m_{1}+m_{2}+m_{3}=6, \quad \quad \lambda=4101,7 \AA$

\begin{tabular}{|c|c|c|c|c|c|c|c|c|}
\hline \multirow{3}{*}{$Z$} & \multicolumn{4}{|c|}{ Berechnet } & \multicolumn{4}{|c|}{ Gemessen } \\
\hline & $m_{3}-$ & $=2 l$ & $m_{3}-n$ & $=2^{l}+1$ & $p-\mathrm{K}$ & omp. & $s-\mathrm{K}$ & mp. \\
\hline & $\Delta \lambda$ & $Q_{m}$ & $\Delta \lambda$ & $Q_{m}$ & $d \lambda$ & Int. & $\Delta \lambda$ & Int. \\
\hline 32 & 38,1 & * & & $y$ & 37,5 & $1(x)$ & & \\
\hline 30 & & & {$[35,8]$} & 5 & & & 34,8 & $1(\mathrm{fr})$. \\
\hline 28 & 33,4 & 4 & & & 33,4 & 7,2 & & \\
\hline $26^{\circ}$ & & & 30,9 & $*$ & & & 30,4 & 1,3 \\
\hline 24 & 28,6 & 4 & & & 28,6 & 2,8 & & \\
\hline 22 & & & 26,2 & 3 & & & 25,8 & 2,4 \\
\hline 20 & 23,8 & 4 & & & 24,2 & 1,1 & & \\
\hline 18 & & & 21,3 & 3 & & & 21,2 & 2,0 \\
\hline 16 & 19,1 & 3 & & & 19,6 & 1,2 & & \\
\hline 14 & & & 16,7 & 3 & & & 17,2 & 1 \\
\hline 1.2 & 14,3 & 3 & & & 14,4 & 1,5 & & \\
\hline 10 & & & 11,9 & (3) & & & 11,9 & 2,1 \\
\hline 8 & 9,5 & 2 & & & 9,6 & 1,2 & & \\
\hline 6 & & & 7,3 & (3) & & & 7,4 & 3,2 \\
\hline 4 & 4,8 & (4) & & & 5,2 & 1 & & \\
\hline 2 & & & 2,4 & (3) & & & 2,4 & 1,3 \\
\hline 0 & 0 & (4) & & & - & & & \\
\hline
\end{tabular}

Sternchen (*) sind die Komponenten bezeichnet, die ihre Entstehung Pendelbahnen (als Anfangsoder Endbahn) verdanken. Wir haben schon erwähnt, daß das Auftreten solcher Bahnen sehr unwahrscheinlich ist; man sieht, dab sie durchweg die schwächsten Komponenten ergeben, und bei den Linien $H_{\alpha}$ und $H_{\gamma}$ zum größten Teil überhaupt nicht aufgenommen werden konnten. Von diesen Komponenten abgesehen, gibt és nur eine einzige theoretisch vorausgesehene, welche sich in den Starkschen Aufnahmen nicht findet $\left(B_{\delta}, Z\right.$ $=0$ ). Dagegen treten drei Linien auf, welche den Quantengleichungen (39) widersprechen, das sind die eingeklammerten $\Delta \lambda$ der Tabellen III und IV. Interessant ist, daß die zwei ersten Ungleichungen streng gelten und in keinem Falle durchbrochen werden, während die dritte, welche sich nach (36) auf den Azimut $\varphi$ bezieht, wie es scheint, nur im groben richtig ist. Eine ähnliche Sonderstellung der azimutalen Quantenzahl konnte Sommerfeld am Bilde der Feinstrukturen bei Funkenerregung des Leuchtens feststellen.

Für die Polarisationen ergibt sich eine sehr merkwürdige empirische Regel: Eine geradzathlige Differenz der azimutalen Quantensahlen $m_{3}-n_{3}$ führt auf" parallele (p-) Polarisation, eine ungeradzahlige auf senkrechte ( $\left.s^{-}\right)$Polarisation. Diese Regel gilt ausnahmslos, wenn uns auch jeder Anhaltspunkt zu ihrem Verständnis fehlt. Es seheint sogar, daß es nicht möglich sein wird, den Polarisationszustand aus den Orientierungen der Anfangs- und Endbahn zum elektrichen Feld za erklären; denn beim Übergang des Elektrons zwischen zwei der vorhin erwähnten Kreisbahnen, die ja immer senkrecht zum Felde stehen, ergeben nach dieser Regel die Linien $H_{\alpha}$ und $H_{\gamma}$ $s$-Komponenten, $H_{B}$ und $H_{\delta} p$-Komponenten.
Für das Zustandekommen einer großen Intensität einzelner Komponenten glaube ich eino notwendige (aber nicht hinreichende) Bedingung darin zu erblicken, daß eine der Quantendifferenzen $m_{1}-n_{1}, m_{2}-n_{2}, m_{3}-n_{3}$ möglichst groB sein muß. Aus diesem Grunde ist die größte dieser drei Differenzen unter $Q_{m}$ in den Tabellen angegeben.

Jedenfalls bilden die besprochenen Ergebnisse der Theorie des Starkeffekts, neben der Sommerfeldschen Theorie der Feinstrukturen, einen der schlagendsten Beweise für die Richtigkeit der Planckschen Quantenlehre und der von Bohr gemáchten Anwendung derselben auf die Atomistik.

IV. Abschnitt.

\section{Struhtur des Phasenraumes.}

\section{\$16. Bedeutung der Plancleschen Hypothese} für die Statistite. - In der Darstellung der Anwendungen haben wir an die von Sommerfeld aufgestellte ( $\$ 11$ ), von Schwarzschild und dem Verfasser präzisierte (\& 12) Fassung der Quantenbedingungen angeknüpft. Wir wollen jetzt auf die Form eingehen, in welcher Planck selbst diese Bedingungen für den Fall mehrerer Freiheitsgrade ausgesprochen hat, und zu diesem Zwecke zunächst die in $\S 3$ gemachten Angaben über die „Hypothese der Wirkungsquanten" durch einige Betrachtungen uber ihre statistische Bedeutung ergänzen.

Der Grund, welcher Planck bewog, von der Auffassung der Energiequanten (\$ 2) abzugehen, war ein Mißverhältnis zwischen dem elektrodynamischen und statistischen Teil der ersten Fassung seiner Theorie der schwarzen Strahlung. Während die Wechselwirkung zwischen Resonatoren und Strahlungsfeld nach den Gesetzen der Elektrodynamik vor sich gehen sollte, und daher ein Resonator jeden beliebigen Energiewert annehmen konnte, wurde bei den Betrachtungen über die Verteilung der zur Verfügung stehenden Energie auf die einzelnen Resonatoren die Annahme gemacht, daf nur diskrete Energiestufen, nämlich Multipla von $h v$, möglich seien.

An Hand der Fig. 1 können wir uns diesen Sachverhalt graphisch veranschaulichen: Wir wissen ( $\$ 3$ ), daß durch Angabe der Lage des Elektrons $x$ (bzw. q) und des Impulses $p$ der Zustand des linearen Resonators vollkommen bestimmt ist. Daher entspricht einem jeden solchen Augenblickszustand, den man nach Gibbs eine "Phase" des Systems nennt, ein Punkt des $(p, q)$-Diagramms oder, wie man sagt, der ,Phasenebenes. Mit der Zeit durchläuft der den $\mathrm{Zu}$ stand eines Resonators repräsentierende Punkt. eine Kurve, die "Phasenbahn". welche, falls keine Energiezufuhr stattfindet, eine Ellipse ist ( $\$ 3$ ). Haben wix eine große Anzahl von Resonatoren, sa entspricht einem jeden in jedem Augenblick ein Punkt des Diagramms und die Verteilung dieser Phasenpunkte gibt uns die Energieverteilung, wenn wir bedenken, daß die Energie in einem 
Punkt durch die Bestimmungsstücke (nach $\$ \$ 2,3$ $A=b^{2} / 2 \mu$ ) der durch ihn hindurchgehenden Ellipse der konzentrischen Schar gegeben ist.

Das Ziel der statistisehen Betrachtungen ist es, die Dichte, mit welcher die Punkte im Diagramm verteilt sind, oder die „Phasendichte“, zu ermitteln, und zwar für den wahrscheinlichsten $\mathrm{Zu}$ stand des aus allen Resonatoren bestehenden Gesantsystems, bei welchem sich die Resonatoren untereinander und mit der Strah]ung im. Gleichlgewicht befinden.

Nach dem ersten elektrodynamischen Teil dex Untersuchung kann nun jeder Energiewert vorkommen und deshalb müßte die Phasendichte hontinuierlich sein, d. h., es müßten in jeder Entfernung vom Nullpunkt Phasenpunkte liegen können. Auf der Mechanik und Elektrodynamik aufgebaute Wahrscheinlichkeitsbotrachtungen sagen uns indessen noch mehr aus: es ist aus ihnen a priori gewiß, daB die Phasendichte eine stetige Funktion des Ortes sein muB, welche auf den elliptischen Kurven konstante Werte annehmend, sich stetig vom Nullpunkt nach außen hiv ändert. Der genauere Verlauf dieser Funktion sol] gerade im zweiten statistischen Teil aufgefunden werden. Aber in diesem war Planck gezwungen, anzunehmen, daß die Punkte in der Phasenebene diskontinuierlich verteilt sind und nur auf den diskreten, der Beziehung $A=h v$ genügenden (in Fig. 1 eingezeichneten) Kurven liegen können. Denn nur so war es möglich, zum richtigen Strahlungsgesetz zu gelangen.

Um diese Härten $z u$ beseitigen und gleichzeitig die nötig gewordene Abänderung der Elektrodynamik auf ein Minimum zu beschränken, änderte Planck seine Hypothese insofern ab, als er annahm, daB zwar die Absorption des linearen Resonators nach den uns bekannten Gesetzen erfolgt, derselbe jedoch im allgemeinen nicht strahlen kang, d. h. daß er keine „Dämpfung" besitzt.

Auf dem Gebiete der Emission läbt sich unser Vorstellungsvermögen einen quantenhaften Prozeß viel eher gefallen; es wird daher angenommen, daß Ausstrahlung nur in den Momenten stattfinden kann (aber nicht stattfinden muB), wenn die Energie des Resonators ein ganzes Vielfaches von $h v$ beträgt. Ur $d$ zwar soll er dann auf einmal seinen gesamten Energieinhalt abgeben. Da jetzt die Elektrodynamils von vornherein aufgegeben wird, so ist anch die SchluBfolgerung auf stetige Anderung der Phasendichte nicht mehr zwingend, aber mit Rücksicht auf die Absorptionsverhältnisse $m u B$ sie nach wie vor kontinuierlich sein. Statt der Annahme der diskontinuierlichen Verteilung der Wahrscheinlichkeit führt Planck deshalb die neve ein, daßs sie zwar kontinuierlich, aber unstetig verteilt ist: Tnnerhalb der ringförmigen Gebiete vom Flächeninhalt $\Delta g=h$, welche durch die ausgezeichneten Kurven der Fig. 1 begrenzt werden, soll die Wahrscheinlichkeit oder, was dasselbe ist, die Phasen- dichte konstant sein und nur an den Grenzkurven selbst zum nächsten Gebiet treppenartig abiallen (bzw. ansteigen). Für die sehwarze Strahlung ergibt sich unter diesen Voraussetzungen dieselbe spektrale Verteilung wie unter den trüheren.

Das Wirkungsquantum $h$ erhält somit die Bedeutung des numerischen Betrages eines Elementarbereiches der Wahrscheinlichkeit, insofern als die Statistik zwischen verschiedenen Punkten eines solchen Bereiches $\Delta_{g}$ nicht unterscheidet.

Die „statischen“ Phasenbahnen repräsentieren nach der neuen Auffassung nicht mehr die einzig möglichen Bewegungen, sondern sind nur insofern ausgezeichnet, als sie die Begrenzungen dex Elementarbereicho darstellen. Eine interessante Folgerung aus diesen Hypothesen besteht darin, daß man den Resonatoren ihre Energie nicht vollständig entziehen kann, indem man sio mit Strahlung von sehr geringer Intensität (d. h. sehr tiefer Temperatur) ins Gleichgewicht setzt. Denn wenn sich alle Phaseupunkte, von welchen jeder einen Resonator darstellt, im innersten Elementarbereich befinden, so ist weitere Energieverminderung nicht möglich, da nach obigem Ausstrahlung nur für Energiewerte $A=n h v$ (d. h. von den statischen Kurven aus) stattfindet. Daher besitzt das System auch im absoluten Nullpunkt der Temperatur eine sogenannte Nullpunktsenergie rom durchschnittlichen Betrage $h \mathrm{v} / 2$ pro Resonator.

Geht man von dem linearen Resonator zur Betrachtung anderer Systeme über, so liegen die Verhältuisse ganz analog: immer kann man die statischen Bahnen entweder als die einzigen möglichen auffassen oder aber die Existenz aller mit der Mechanik verträglichen Bahnen zulassen und dis statischen nur als wahrscheinlichkeitstheoretisch ausgezeichnete betrachten. Die leiztere Auffassung, deren Vertreter Planck ist, hat in keinem Falle zu Widersprüchen mit der Erfahrung geführt, vielmehr kann man vielleicht von ihr die Aufklärung einzelner, bis jetzt dunkler Probleme erwarten (spezifische Wärme zweiatomiger Gase). Eine scheinbare Schwierigkeit, die sich in einem Spezialfall (Theorie des Rotationspektrums) ergab, hat Planck neuerdings in überraschender Weise hinweggeräumt. Aucb die Bohrsche Theorie $(\$ \S 7,8$ ) läbt sich mit diesem Standpunkt ohne weiteres vereinbaren, wenn man die Bedeutung der Bohrschen Frequenzbedingung etwas verschiebt: Man 'braucht nur' anzunehmen, daB Strahlung (bzw. Energieänderung) normalerweise nicht, stattfindet, wenn aber das Elektron gestört wird, dasselbe in eine neve Bahn überspringt, wobei die ausgesandte Schwingungszahl nach der Frequenzbedingung (16) durch die Energien der Grenzen des alten und neuen Elementarbereiches bestimmt wird.

\& 17. Struktur des Phasenraumes. - Im Lichte der Ausführungen des letzten Paragraphen lassen sich die Standpunkte, welche den beiden. 
Fassungen (5) und (5̌a) bezw. (5b) der Planckschen Quantenbedingung zugrunde liegen, wie folgt präzisieren: Im ersten Falle wird das Doppelintegral über Phásenpunkte, welche verschiedenen Energieinhalten eines Resonators entw sprechen, ausgedehnt, d. h. die statischen Kurven, werden aus einer Betrachtung aller Zustände, welche der Resonator unter ganz verschiedenen Bedingungen annehmen kann, erschlossen. Im zweiten erstreckt sich die Integration über eine individuelle, ohne äußere Störung bei konstanter Energie verlaufende Bewegung des Resonators. Dementsprechend kann man auch bei der Behandlung mehrdimensionaler Systeme an die eine oder die andere Auffassung anknüpfen. Den zweiten Weg hat Sommerfeld beschritten ( $\$ 11)$, der erste wurde gleichzeitig won Planck eingeschlagen.

Im allgemeinen Fall von $f$ Freiheitsgraden mit den Variablen $q_{i}$ und Impulsen $p_{i}$ $(i=1,2 \ldots f)$ entspricht einem Augenblickszustand oder einer, Phase" des Systems ein spezielles Wertesystem der $2 f$ Bestimmungsstücke $p_{i}, q_{i}$. Die Gesamtheit aller Phasen bildet nicht eine Ebene, sondern eine Mannigfaltigkeit von of Dirmensionen; einen idealen $2 f$-dimensionalen "Phasenraum", wenn man die $p_{i}, q_{i}$ als rechtwinkelige Foordinaten auffaßt. Auch hier fragt Planch nach den Elementargebieten $\Delta G$ der Wahrscheinlichkeit oder nach der ,Struktur des Phasenraumes". Da jedes einzelne Produkt $p_{i} \cdot q_{i}$ schon die Dimension einer Wirkung hat, so muB der Rauminhalt des Elemeutargebietes (von $2 f$ Dimensionen)

$$
A G=h^{\mathrm{f}}
$$

gesetzt werden. Wie findet man nun die Begrenzungen der Elementarbereiche $\Delta G$ ? Planck tut dies, indem er $f$ Ausartungen der allgemeinen Bewegung des Sytems aussucht, von denen jede eindimensional verläuft, und die daher nach $\$ 3$ behandelt werden können. Aus den Lösungen dieser Spezialfälle wird dann die allgemèine Lösung aufgebaut.

$\mathrm{Ob}$ es in jedem Falle möglich, $f$ ausgeartete Bewegungen von der geforderten Beschaffenheit anzugeben, ist noch nicht untersucht. Jedoch läBt sich diese Forderung jedenfalls in einer umfassenderen Gruppe von Systemen erfüllen, als es die bedingt periodischen ( $\$ 12$ ) sind; und für diese Tetztere Klasse von Bewegungen lassen sich die Planckschen Vorschriften vollständig durchführen. Z. B. wären bei der Keplerbewegung des S $11(f=2)$ die Kreisbahn ( $r=$ const) und die zll einer Geraden ausartende Ellipse vom Achsenverhältnis Null ( $\varphi=$ const) zu betrachten; beim Starkeffekt $(f=3)$ die drei auf $S .247$ besprochenen Ausartungen (Kreisbahn und die beiden Pendelbahnen). Man kann nun allgemein zeigen, daB fü ein bedingt periodisches System der Ausdruck für den Rauminhalt des Elementarbereichs im allgomeimen in $f$ Faktoren zerfällt.

$$
A G=\Delta g_{1} \cdot \Delta g_{2} \ldots A g_{f} \text {, }
$$

von denen jeder $\left(\Delta g^{\circ}\right)$ den Flächeninhalt eines Be- reiches der entsprechenden Koordinatenebene $\left(p_{i}, q_{i}\right)$ vom Betrage $h$ darstellt; ferner, daß die quantentheoretisch ausgezeichneten (statischen) Bewegungen des Systems genau mit den durch die Bedingungen (29), $\left(29^{\prime}\right)$ des $\$ 12$ festgelegten zusammenfallen ${ }^{1}$ ). Demnach waren in der Plancksehen Theorie die Quantenbedingungen (89) für bedingt periodische Systeme, nebst den aus innen in den \$\& 13 und 15 für Spezialfälle gezogenen Schlüssen bereits implizite (obwohl, wie es scheint, bis jetzt nur teilweise erkannt) enthalten.

Auf eine von Einstein') gegebene interessante Fassung derselben Bedingungen können wir im Rahmen dieses Aufsatzes nicht eingehen, wir wollen jedoch an dieser Stelle einen wichtigen Gesichtspunkt, der an die Auffassung, dab die statischen Bahnen die einzigen möglichen sind, anknüpft und von Whrenfest (1916) herrührt, kurz erwähnen. Man betrachte die Anderung, welche ein System erfährt, wenn man einen äuBeren Parameter desselben (d. h. nicht eine der Variablen $p, q$ ) langsam beeinflubt, z. B. wenn man im Starkeffekt das äußere elektrische Feld a]Imählich anwachsen läßt. Einen solchen Prozeb nennt man eine ,adiabatische Zustandsänderung", weil dabei die Energie des Systems nur durch Vermittlung des äuBeren Parameters, nicht aber durch direkte Zuführung lebendiger Kraft verändert wird; genau wie bei der adiabatischen Kompression eines Gases eine Änderung des Energieinhalts nur durch die aufgewandte Arbeit und nicht durch direkten Wärmeaustausch erfolgt. Im Anfangszustand führt das System nach der Quantentheorie ingend eine statische Bewegung aus; geht man von einer solchen aus, und unterwirft dasselbe einem unendlich langsamen adiabatischen ProzeB, so kann man einerseits die Quantenbedingungen vorübergehend auber Acht lassen wad nach der Bewegungsform fragen, welche aus der ursprünglichen rein mechanisch hervorgeht. Denn die Anfangsbewegung erfährt bei diesem Prozeß nach den Gesetzen der Mechanik eine stetige Veränderung, so dab ihr ganz eindentig in jedem Augenblick eine neve entspricht. Andererseits könnte man für jeden Wert des Parameters die Quantenbedingungen aufstellen und die mit ihnen verträglichen statischen Bahnen ermitteln. Es entsteht nun die Frage, of Mechanik und Quantentheorie sich ununterbrochen gegenseitig stören und durchkreuzen. oder ob sie übereinstimmend auf dieselben Bewegungsformen führen? Der Inhalt der Ehrenfestschen Adiabatenhypothese beteht darin, das das Letztere angenommen wird: Quantentheoretisch zulässige Bewegungen gehen durch einen unendlich langsamen adiabatischen ProzeB

1) Nach unveröfientlichten Untersuchungen des Verfassers. In gewissen Spezialfällen muk man von beidea Standpunkten aus an Stelle ron $p$ in (29) $p-p_{0}$ (vgl. \$ 3) schreiben. $191 \%$.

${ }^{2}$ A. Einstein, Verh. d. D. phys. Ges, 19, S. 82 , 
(rein mechanisch) wieder in quantentheoretisch zulässige über. Hieraus folgt, daß diejenigen Konstanten der Bewegung, welche nach den Quantenbedingungen (25) 'der Universalkonstanten $n, h$ gleich gesetzt werden, sich während einer adiabatischen Zustandsänderung nicht ändern können oder, wie man sich ausdrückt, ,adiabatisch invariant" sind. Die Prüfung dieser Hypothese für periodische (Ehrenfest) und bedingt periodische (Burgers) Systeme ergab, daß die Quantenintegrale der $\$ \$ 11$ und 12 in der Tat adiabatische Invarianten darstellen.

Die Adiabatenhypothese hat bereits zur Aufklärung gewisser begrifflich schwieriger Fälle beigetragen; ihre Wichtigkeit liegt auf der Hand, wenn man bedenkt, daß man durch adiabatische Prozesse von einfachen Systemen zu komplizierteren übergehen kann. Natürlieh kann man diese Hypothese auch auf den andern Standpunkt, da s die statischen Bewegungen nicht die einzig möglichen, sondern nur wahrscheinlichkeitstheoretsich ausgezeichnet sind, übertragen, indem man in ihrer Formulierung das Wort ,zulässige" durch ,ausgezeichnete" ersetzt.

\$ 18. Schluß. - Durch die vorangehenden Ausführungen; besonders des II. und III. Abschnittes, wird der Leser den Eindruck gewonnen haben, dab die Plancksche Theorie auf dem noch jungen Gebiet ihrer Anwendungen auf die Atomistik und Spektroskopie bereits eine Reihe gesicherter Ergebnisse aufzuweisen hat, welche man ohne Ubertreibung als große Erfolge bezeichnen kann, und welche den Versuch rochtfertigen, diesen Gegenstand einem größeren Loserkreise zugänglich zu machen. Jeder, der auf diesem Gebiete arbeitet, ist sich indessen bewußt, daB wir noch weit davon entfernt sind, überall klar zu sehen: Noch viele begriffliche und rechnerische Sohwierigkeiten müssen überwunden, viele provisorisehe Vorstellungen verschoben oder ersetzt werden.

Zunächst wird man die Frage stellen, wie man bei allgemeineren mechanischen Systemen als die bedingt periodischen die quantentheoretisch zulässigen Bewegungen auffinden kann. Ansätze zu. ihrer Beantwortung sind bereits vorhanden: Wir haben bereits erwähnt, daß die Plancksche Behandlungsweise über den Bereich des bedingt Periodischen hinausgreift, wenn es auch nicht leicht sein dürfte, zu den eindeutigen Vorschriften für ihre allgemeine Durchführung zu gelangen. Andererseits hat Burgers ${ }^{1}$ ) auf eine von Delaunay (1860) in die Himmelsmechanik eingeführte $\mathrm{Me}-$ thode hingewiesen, deren begrifflicher Inhalt darin besteht, ein System durch eine Reihe nach einem bestimmten Verfahren sukzessive auszuwählender bedingt periodischer Systeme beliebig weit zu approximieren. Der Verfasser war bereits ohne Kenntnis der Delaunaysehen Unter1917

1) J. M. Burgers, Amsterdam Proceedings, S. 170, suchung bei der Behandiung spezieller Beispiele auf die Möglichkeit einer solchen Approximation aufmerksam geworden. Die von ihm nach diesem Verfahren ausgeführten Berechnungen über das Spektrum des neutralen Heliums (Dreikörperproblem) scheinen aber leider zu keinen großen Hoffnungen auf die Methode zu berechtigen. Schließlich dürfte es möglich sein, die im letzten \& besprochene Adiabatenbypothese von Ehrenfest als heuristisches Mittel zu verwenden.

Dies sind indessen rein praktische Fragen, deren Lösung uns vielleicht schon die allernächste Zukunft bringen wird. Ernster jedoch scheinen die Schwierigkeiten zu sein, welche mit der prinzipiellen Seite der von Bohr gemachten Annahmen $(\$ \& 7,8)$ zusammenhängen. Wir haben an verschiedenen Stellen erwähnt, das sich die Quantentheorie in bewußten Gegensatz zur Elektrodynamik setzt und der letzteren im Atominnern nur eine beschränkte Gültigkeit zugesteht. Wie muß man aber die Elektrodynamik abändern, um diese Gegensätze auszugleichen? Daß es eine Synthese der beiden Lehren ist; welche uns fehlt, sieht man u. a. aus dem folgenden Umstand: Der Starkeffekt wird durch die Quantentheorie vollständig erklärt, während die Elektrodynamik für ihn vereagt; umgekehrt ist es bis jetzt bei der scheinbar verwandten Erscheinung des Zeemaneffekts (Aufspaltung von Spektrallinien im Magnetfelde), hier komme man vorläufig mit der Elektrodynanik weiter als mit dex Quantentheorie (wenn auch zu keiner vollständigen Erklärung). Warum findet während der Bewegung in einer statischen Bahn keine Strahlung statt, und wie liegen die Verhältnisse, während das Elektron von einer statischen Bahn zu einer andern springt? Die einzigen Tatsachen, welche uns über den letzteren Vorgang bekannt sind, sind die Bohrsche Frequenzbedingung ( $\$$ ) und die Polarisationsregel beim Starkeffekt $(\& 15)$. Und dieso beiden Gesetze tragen in ihrer heutigen Fassung einen gewissermaßen teleologischen, den Naturforscher geradezu verletzenden Charakter, so dab viele Fachgenossen sich an diesen "Bauernregeln" mit Recht stoßen. Es wäre in der Tat sehr erwünscht. den Emissionsvorgang in allen Finzelheiten zu überblicken und die Erklärung der erwähnten Gesetze einzusehen. Wenn diese Forderungen für den Augenblick auch zu hoch gespannt erscheinen, so könnte man vielleicht schon jetzt mit mehr Aussicht auf Erfolg versuchen, durch Einbeziehen der Freiheitsgrade des Äthers die Frequenzbedingung auf dieselbe Form (25) zu bringen, wie die für die Materie gültigen Quantenbedingungen.1)

Wenn demnach die Quantentheorie noch viele

1) Wie A. Rubinowics (Plyys. Zs. 18, S. 96, 1917) gezeigt hat, läBt wich dies fir die Theorie der schwarzen Strablung durchfuhren; auch die im letzten Satz des Textes enthaltene Bemerkung verdanke ich Herro Rubinowice. 
Lücken auszufüllen hat, vielleicht sogar erst in den Anfängen steckt, können wir immerhin mit dem Tempo des Anwachsens der Kenntnisse vom Atom, welche wir ihr verdanken, zufrieden sein. Wer den Gang der Entwieklung in der letzten Zeit und die erreichten Erfolge ohne Voreingenommenheit überblickt, muß zugeben, daß das Werk von Max Planch uns auf dem Wege zum letzten Ziel der exakten Naturwissenschaften, der Erkenntnis der Struktur von Materie und Äther, um eine Strecke weitergebracht hat, welche noch vor wenigen Jahren auch den kühnsten Hoffnungen in weite, unbestimmte Ferne zu führen schien.

\section{Über den Begriff des Zufalls und den Ursprung der Wahrscheinlich- keitsgesetze in der Physik.}

Von Prof. M. v. Smoluchowski $t$, Krakau.

I.

Die Wahrscheinlichkeitsrechnung, welche seit Beginin ihrer Entwicklung mit größtem Erfolg hauptsächlich in dem sonst der mathematischen Bèhandlung wenig zugänglichen Bereich sozialer und biologischer Vorgänge angewendet wurde, hat sich in den letzten Zeiten ein überaus wichtiges Anwendungsgebiet erobert: die Physik. Und zwar ist damit nicht etwa die seit Gauß' Zeiten als eigene Hilfsdisziplin ausgebildete Theorie der Fehlerausgleichung bei physikalischen Messungen gemeint, sondern gerade das eigentliche Gerüst dieser Wissenschaft, das System der theoretischen Physik.

Zum ersten Male in den Jahren 1857-1860 von Clausius und Maxwell als eigenartiges mathematisches Hilfsmittel in die kinetische Gastheorie eingeführt, hat die Wahrscheinlichkeitsrechnung, nach einer vorübergehenden Periode der Stagnation, infolge des schlieBlichen Sieges der atomistischen Anschauungsweise eine für die Physik ganz grundlegende Bedeutung gewonnen und bildet heute das wichtigste Werkzeug bei Forschungen auf dem Gebiete der modernen Theorien der Materie, der Elektronik, Radioaktivität und Strahlungstheorie. Entspricht doch ihr Wesen durchaus der heute zur Herrschaft gelangten Tendenz, sämtliche Gesetze der Physikㄱ) nach dem Vorbild der kinetischen Gastheorie auf Statistik verborgener Elementarereignisse zurückzuführen, wobei die „Einfachheit" derselben als sekundäre Folge des Wahrscheinlichkeitsgesetzes "der großen Zahlen" aufgefabt wird.

Trotz dieser enormen Ausdehnung des Anwendungsbereiches der Wahrscheinlichkeitsrech-

1) Von dieser Tendenz sind bisher nur die Torentzschen Gleichungen der Elektronentheorie, das Energiegesetz und Relativitätsprinzip unberührt geblieben, aber es ist wohl möglich, daB im Laufe der Z̈eit auch hier exakte Gesetzesformen 'durch statistische RegelmäBigkeit ersetzt werden düriten. nung hat die exalte Analyse der ihr zugrunde liegenden Begriffe nur geringe Fortschritte gemacht; es gilt wohl noch hente der Satz, daB keine zweite mathematische Disziplin auf so unklaren und schwankenden Grundlagen aufgebaut ist. So werden die Grundfragen nach der Subjektivität oder Objektivität des Wahrscheinlichkeitsbegriffes, nach der Definition der Zufälligkeit usw. von verschiedenen Autoren in diametral entgegengesetzter Weise beantwortet. Insbesondere ist auch eine allgemeine und mathematisch exakte Präzisierung der für die Anwendbarkeit dieser Rechnungsmethode charakteristischen Bedingungen noch immer ausständig, und man pflegt sich in dieser Hinsicht meist auf ein intuitives Wahrscheinlichkeitsgefühl zu verlassen.

Als kleiner Beitrag zu derartigen Uatersuchungen mögen die nachfolgenden Bemerkungen aufgefaßt sein, welche von der Anwendung der Wahrscheinlichkeitsrechnung in der Physik ausgehen, in der gewisse grundsätzliche Schwierigkeiten in besonders krasser Form auftreten. Ich will eingestehen, daß gerade das Unbefriedjgende der diesbezüglichen Ausführungen in gewissen, sonst höchst beachtenswerten neueren Werken die Entstehung dieser Studie veranlaBt hat. Im übrigen bezweckt dieselbe selbstverständlich keineswegs eine allseitige und endgültige Aufklärung des ganzen damit zusammenhängenden Komplexes philosophischer Fragen, sondern will nur eine Anregung zu weiteren Untersuchungen in einer bestimmten Richtung geben, indem einige Leitgedanken hervorgehoben werden, welche die bisher allzusehr vernachlässigte objektive Seite des Wahrscheinlichkeitsbegriffes ins rechte Licht setzen sollen.

\section{II.}

Die Frage, welche Ereignisse in den Geltungsbereich der Wahrscheinlichkeitsrechnung fallen, wird wohl allgemein dahin beantwortet: diejenigen, deren Eintritt vom Zufall abhängt. Die Untersuchung dieses letzteren Begriffes ist also jedenfalls das Primäre, und wir werden uns vor allem klar zu machen suchen, wodurch das Wesen des Zufalls gekennzeichnet ist. Damit hängen $z$ wei vielumstrittene Probleme zusammen, deren Schwierigkeit angesichts der exakten mathematischen Spekulationen der theoretischen Physik sich besonders fühlbar macht, nämlich:

1. Wie ist es möglich, dab sich der Effekt des Zufalls berechnen lasse, daß also $z u-$ fällige Ursachen gesetzmäßige Wirkungen haben?

2. Wie kann der Zufall entsteben, wenn alles Geschehen nur auf regelmäBige Naturgesetze zurückzuführen ist? oder mit anderen Worten: Wie können gesetzmäßige Ursachen eine zufällige Wirkung haben?

Betrachtet man in populärer Weise den $\mathrm{Zu}$ fall als die Negation des Gesetzmäßigen, so sind diese Widersprüche gewiß vollständig unüber- 